\title{
Duality invariant cosmology to all orders in $\alpha^{\prime}$
}

\author{
Olaf Hohm ${ }^{1}$ and Barton Zwiebach ${ }^{2}$ \\ ${ }^{1}$ Institute for Physics, Humboldt University Berlin, Zum Großen Windkanal 6, D-12489 Berlin, Germany \\ ${ }^{2}$ Center for Theoretical Physics, Massachusetts Institute of Technology, \\ Cambridge, Massachusetts 02139, USA
}

(Received 14 June 2019; published 10 December 2019)

\begin{abstract}
While the classification of $\alpha^{\prime}$ corrections of string inspired effective theories remains an unsolved problem, we show how to classify duality invariant $\alpha^{\prime}$ corrections for purely time-dependent (cosmological) backgrounds. We determine the most general duality invariant theory to all orders in $\alpha^{\prime}$ for the metric, $b$-field, and dilaton. The resulting Friedmann equations are studied when the spatial metric is a timedependent scale factor times the Euclidean metric and the $b$-field vanishes. These equations can be integrated perturbatively to any order in $\alpha^{\prime}$. We construct nonperturbative solutions and display duality invariant theories featuring string-frame de Sitter vacua.
\end{abstract}

DOI: $10.1103 /$ PhysRevD.100.126011

\section{INTRODUCTION}

String theory is arguably the most promising candidate for a theory of quantum gravity. As a theory of gravity, the prospect for a confrontation between string theory and observation seems to be particularly promising in the realm of cosmology, where the effects of fundamental physics at very small scales may be amplified to very large scales. Since the early days of string theory there have been intriguing ideas of how its unique characteristics could play a role in cosmological scenarios [1-3], ideas that have been revisited and extended recently [4-7]. Two such features of classical string theory will be central for the present paper: (i) the existence of dualities that for cosmological backgrounds send the scale factor $a(t)$ of the universe to $1 / a(t)$, in sharp contrast to Einstein gravity, and (ii) the presence of infinitely many higher-derivative $\alpha^{\prime}$ corrections.

It is a natural idea that the higher-order corrections of string theory play a role, for instance, in resolving the bigbang singularity. The first step is the inclusion of the $\alpha^{\prime}$ corrections of classical string theory. Such corrections have been computed to the first few orders in the 1980s [8-10], but a complete computation or classification of these corrections is presently out of reach. In recent years duality-covariant formulations of the string spacetime theories (double field theory [11-14]) have been used to make progress in describing $\alpha^{\prime}$ corrections; see [15-21]. These developments hint at a completely novel kind of

Published by the American Physical Society under the terms of the Creative Commons Attribution 4.0 International license. Further distribution of this work must maintain attribution to the author(s) and the published article's title, journal citation, and DOI. Funded by SCOAP ${ }^{3}$. geometry where the diffeomorphism invariance of general relativity is replaced by a suitably generalized notion of diffeomorphisms, which in turn partly determines the $\alpha^{\prime}$ corrections. These ideas play a key role in the "chiral" string theory of [15], which is the only known gravitational field theory that is exactly duality invariant and has infinitely many $\alpha^{\prime}$ corrections. This program, however, has not yet been developed to the point that it can deal with the set of all $\alpha^{\prime}$ corrections for bosonic or heterotic string theory. (See, however, the recent proposal for heterotic string theory [22].)

This state of affairs is unsatisfactory since the inclusion of a finite number of higher-derivative $\alpha^{\prime}$ corrections is generally insufficient. Gravitational theories with a finite number of higher derivatives typically display various pathologies that are an artifact of the truncation and not present in the full string theory [23]. In this paper we will bypass these difficulties by classifying the higher-derivative corrections relevant for cosmology to all orders in $\alpha^{\prime}$. Rather than finding the complete $\alpha^{\prime}$ corrections in general dimensions and then assuming a purely time-dependent cosmological ansatz, we immediately consider the theory reduced to one dimension (cosmic time) and determine the complete higher-derivative corrections compatible with duality. String theories, being duality invariant, must correspond to some particular points in this theory space. While we do not know which points those are, the full space of duality invariant theories is interesting in its own right, and may exhibit phenomena that are rather general and apply to string theory.

Our analysis is based on the result by Veneziano and Meissner [24], extended by Sen [25], concerning the classical field theory of the metric, the $b$-field, and the dilaton arising from $D=d+1$ dimensional string theory. 
This field theory displays an $O(d, d, \mathbb{R})$ symmetry to all orders in $\alpha^{\prime}$ provided the fields do not depend on the $d$ spatial coordinates. This symmetry, henceforth referred to as "duality," contains the scale-factor duality $a \rightarrow a^{-1}$. The work of Meissner [26] implies that in terms of standard fields the duality transformations receive $\alpha^{\prime}$ corrections, but it was shown that to first order in $\alpha^{\prime}$ there are new field variables in terms of which dualities take the standard form. We will assume that there are field variables so that duality transformations remain unchanged to all orders in $\alpha^{\prime}$ (this certainly happens in conventional string field theory variables [27]). With this assumption we are able to classify completely the duality invariant $\alpha^{\prime}$ corrections. This work amounts to an extension and elaboration of the results obtained by us in [28]. We use the freedom to perform duality-covariant field redefinitions to show that only firstorder time derivatives need to be included and that the dilaton does not appear nontrivially, thereby arriving at a minimal set of duality invariant higher-derivative terms to all orders. We prove that at order $\alpha^{\prime k}$ the number of independent invariants, and thus the number of free parameters not determined by $O(d, d, \mathbb{R})$, is given by $p(k+1)-p(k)$, with $p(k)$ the number of partitions of the integer $k$.

Let us briefly summarize the core technical results of the first part of the paper. The two-derivative spacetime theory for the metric $g$, the $b$-field $b$, and the dilaton $\phi$, restricted to depend only on time, is described by the one-dimensional action [24]

$$
I_{0}=\int \mathrm{d} t e^{-\Phi}\left(-\dot{\Phi}^{2}-\frac{1}{8} \operatorname{tr}\left(\dot{\mathcal{S}}^{2}\right)\right),
$$

where $\Phi$ is the $O(d, d, \mathbb{R})$ invariant dilaton, defined by $e^{-\Phi}=\sqrt{\operatorname{det} g} e^{-2 \phi}$, and we introduced the $O(d, d, \mathbb{R})$ valued matrix

$$
\mathcal{S} \equiv\left(\begin{array}{cc}
b g^{-1} & g-b g^{-1} b \\
g^{-1} & -g^{-1} b
\end{array}\right),
$$

in terms of the spatial components $g$ and $b$ of the metric and $b$-field. Our classification implies that the most general duality invariant $\alpha^{\prime}$ corrections take the form

$$
\begin{aligned}
I \equiv & I_{0}+\int \mathrm{d} t e^{-\Phi}\left(\alpha^{\prime} c_{2,0} \operatorname{tr}\left(\dot{\mathcal{S}}^{4}\right)+\alpha^{\prime 2} c_{3,0} \operatorname{tr}\left(\dot{\mathcal{S}}^{6}\right)\right. \\
& +\alpha^{\prime 3}\left[c_{4,0} \operatorname{tr}\left(\dot{\mathcal{S}}^{8}\right)+c_{4,1} \operatorname{tr}\left(\dot{\mathcal{S}}^{4}\right) \operatorname{tr}\left(\dot{\mathcal{S}}^{4}\right)\right] \\
& +\alpha^{\prime 4}\left[c_{5,0} \operatorname{tr}\left(\dot{\mathcal{S}}^{10}\right)+c_{5,1} \operatorname{tr}\left(\dot{\mathcal{S}}^{6}\right) \operatorname{tr}\left(\dot{\mathcal{S}}^{4}\right)\right] \\
& +\alpha^{\prime 5}\left[c_{6,0} \operatorname{tr}\left(\dot{\mathcal{S}}^{12}\right)+c_{6,1} \operatorname{tr}\left(\dot{\mathcal{S}}^{8}\right) \operatorname{tr}\left(\dot{\mathcal{S}}^{4}\right)+c_{6,2}\left(\operatorname{tr}\left(\dot{\mathcal{S}}^{6}\right)\right)^{2}\right. \\
& \left.+c_{6,3}\left(\operatorname{tr}\left(\dot{\mathcal{S}}^{4}\right)\right)^{3}\right]+\cdots,
\end{aligned}
$$

where only first-order time derivatives of $\mathcal{S}$ need to be included. Moreover, there are no terms involving $\operatorname{tr}\left(\dot{\mathcal{S}}^{2}\right)$.
The pattern is clear: the general term at order $\alpha^{\prime k}$ involves traces with $2 k$ factors of $\dot{\mathcal{S}}$. Each trace must have an even number of $\dot{\mathcal{S}}$ factors, where the even number cannot be two. The $c$ 's are a priori undetermined coefficients, duality holding for any value they may take. Except for a few of them, their values for the various string theories are unknown. In establishing the above result, we made repeated use of field redefinitions iteratively in increasing orders of $\alpha^{\prime}$. The result is striking in that only first derivatives of the fields appear in the action. All duality invariant terms with more than one time derivative on $\mathcal{S}$ can be redefined away. All terms with one or more derivatives of the dilaton can also be redefined away. The resulting higher derivative actions are in fact actions with high numbers of fields acted on by one derivative each. This is a major, somehow unexpected, simplification.

In the second part of this paper we investigate this general $\alpha^{\prime}$-complete theory for the simplest cosmological ansatz, a Friedmann-Lemaitre-Robertson-Walker (FLRW) background whose spatial metric is given by a timedependent scale factor times the Euclidean metric and a vanishing $b$-field. The resulting Friedmann equations are determined to all orders in $\alpha^{\prime}$. While there is of course still an infinite number of undetermined $c_{k, l}$ parameters in (1.3), we can write the equations efficiently in terms of a single function $F(H)$ of the Hubble parameter whose Taylor expansion is determined by these coefficients:

$$
F(H):=4 d \sum_{k=1}^{\infty}\left(-\alpha^{\prime}\right)^{k-1} 2^{2 k-1} c_{k} H^{2 k},
$$

where, in terms of (1.3), $c_{k}:=c_{k, 0}+2 d c_{k, 1}+\cdots$. The Friedmann equations then take the concise form

$$
\begin{aligned}
\frac{d}{d t}\left(e^{-\Phi} f(H)\right) & =0, \\
\ddot{\Phi}+\frac{1}{2} H f(H) & =0, \\
\dot{\Phi}^{2}+g(H) & =0,
\end{aligned}
$$

where the functions $f(H)$ and $g(H)$ are determined in terms of $F(H)$ as

$$
f(H):=F^{\prime}(H), \quad g(H):=H F^{\prime}(H)-F(H),
$$

where ' denotes differentiation with respect to $H$.

We show that solutions of the lowest-order equations given by Mueller in [29] can be extended, perturbatively, to arbitrary order in $\alpha^{\prime}$. We then turn to the arguably most intriguing implication of our results: the potential existence of interesting cosmological solutions that are nonperturbative in $\alpha^{\prime}$. We discuss a general nonperturbative initial-value formulation, and we state conditions on the function $F(H)$ so that the theory permits de Sitter vacua (in string frame). 
There are functions satisfying these criteria, and so there are duality invariant theories with nonperturbative de Sitter vacua, suggesting that string theory may realize de Sitter in this novel fashion [30]. Note that the Lagrangian for the theory does not include a cosmological constant term. We also note that in pure gravity the possibility of generating inflation through restricted higher-derivative interactions leading to second-order Friedmann equations has been investigated [31]. Here we do not constrain the number of derivatives in the original theory. Rather, duality invariance and field redefinitions lead to two-derivative equations in the cosmological setting.

The paper is organized as follows. In Sec. II we review the two-derivative theory and its equations of motion and then tackle the classification problem. We examine carefully the freedom to perform field redefinitions, including those of the lapse function $n(t)$, which is usually set to one by a gauge choice. In Sec. III we derive the equations of motion of the higher-derivative action restricted to the single trace terms, and then compute the Noether charges for the global duality symmetry. Section IV specializes to the FLRW metric with zero curvature and derives the Friedmann equations to all orders. As it turns out, this result is valid even when the action contains the most general multitrace terms. The solutions in perturbation theory of $\alpha^{\prime}$ are determined. In Sec. V we consider nonperturbative solutions. We solve the initial-value problem and show how de Sitter solutions are possible in the space of dualityinvariant theories. We conclude this paper with a discussion of possible further generalizations.

\section{CLASSIFICATION OF COSMOLOGICAL $O(d, d)$ INVARIANTS}

Our goal is to classify $O(d, d)$ invariant actions to all orders in $\alpha^{\prime}$, up to field redefinitions. This was partially done in [28], whose results will here be completed by allowing for $O(d, d)$ covariant field redefinitions of the lapse function.

\section{A. Review of two-derivative theory}

We start with the two-derivative theory for metric $g_{\mu \nu}$, antisymmetric $b$-field $b_{\mu \nu}$, and the scalar dilaton $\phi$,

$I_{0}=\int d^{D} x \sqrt{-g} e^{-2 \phi}\left(R+4(\partial \phi)^{2}-\frac{1}{12} H^{2}-\frac{2(D-26)}{3 \alpha^{\prime}}\right)$,

where $H_{\mu \nu \rho}=3 \partial_{[\mu} b_{\nu \rho]}$. Here we displayed the action for a generally noncritical bosonic string theory in $D$ dimensions. In the following we assume $D=26$ but comment on the general case in Sec. V C below. We now drop the dependence on all spatial coordinates; i.e., we set $\partial_{i}=0$, where $x^{\mu}=\left(t, x^{i}\right), i=1, \ldots, d$, and we subject the fields to the ansatz

$$
\begin{aligned}
g_{\mu \nu} & =\left(\begin{array}{cc}
-n^{2}(t) & 0 \\
0 & g_{i j}(t)
\end{array}\right), \quad b_{\mu \nu}=\left(\begin{array}{cc}
0 & 0 \\
0 & b_{i j}(t)
\end{array}\right), \\
\phi & =\phi(t) .
\end{aligned}
$$

The resulting one-dimensional two-derivative action then takes the $O(d, d)$ invariant form [24]

$I_{0} \equiv \int \mathrm{d} t n e^{-\Phi} L_{0}=\int \mathrm{d} t n e^{-\Phi} \frac{1}{n^{2}}\left(-\dot{\Phi}^{2}-\frac{1}{8} \operatorname{tr}\left(\dot{\mathcal{S}}^{2}\right)\right)$,

where

$\mathcal{S} \equiv \eta \mathcal{H}=\left(\begin{array}{cc}b g^{-1} & g-b g^{-1} b \\ g^{-1} & -g^{-1} b\end{array}\right), \quad \eta=\left(\begin{array}{ll}0 & 1 \\ 1 & 0\end{array}\right)$.

The field $\mathcal{S}$ is constrained: it satisfies $\mathcal{S}^{2}=\mathbf{1}$. We have also defined the $O(d, d)$ invariant dilaton $\Phi$ via

$$
e^{-\Phi}=\sqrt{\operatorname{det} g_{i j}} e^{-2 \phi} .
$$

The action is time-reparametrization invariant, with $n(t)$ transforming as a density under time reparametrizations $t \rightarrow t-\lambda(t)$ :

$$
\delta_{\lambda} n=\partial_{t}(\lambda n)
$$

A scalar $A$ under this reparametrization transforms as $\delta_{\lambda} A=\lambda \partial_{t} A$. The metric, the $b$-field, and the dilaton $\Phi$ are scalars under time reparametrizations. Defining the covariant time derivative $\mathcal{D}$,

$$
\mathcal{D} \equiv \frac{1}{n(t)} \frac{\partial}{\partial t}
$$

one can quickly show that if $A$ is a scalar, so is $\mathcal{D} A$. The covariant derivative satisfies the usual integration by parts rule

$$
\int \mathrm{d} t n B \mathcal{D} A=\int \mathrm{d} t \partial_{t}(A B)-\int \mathrm{d} t n(\mathcal{D} B) A
$$

The action $I_{0}$ above can now be written in a manifestly reparametrization invariant form

\footnotetext{
${ }^{1}$ This result generalizes to the compactification of the $D$-dimensional theory on a $d$-torus to $D-d$ dimensions, which also exhibits a global $O(d, d)$ symmetry [25,33,34].
} 


$$
I_{0}=\int \mathrm{d} t n e^{-\Phi}\left(-(\mathcal{D} \Phi)^{2}-\frac{1}{8} \operatorname{tr}\left((\mathcal{D} S)^{2}\right)\right)
$$

The variation of the dilaton yields the dilaton equation of motion $E_{\Phi}=0$,

$$
\begin{aligned}
\delta_{\Phi} I_{0} & =\int \mathrm{d} \operatorname{tne}^{-\Phi} \delta \Phi E_{\Phi} \\
E_{\Phi} & \equiv 2 \mathcal{D}^{2} \Phi-(\mathcal{D} \Phi)^{2}+\frac{1}{8} \operatorname{tr}\left((\mathcal{D} S)^{2}\right) .
\end{aligned}
$$

Let us explain first in general terms how we vary with respect to $\mathcal{S}$ to find field equations. Consider an arbitrary $\mathcal{S}$ dependent action $I$ of the form

$$
I=\int \mathrm{d} t n e^{-\Phi} \mathcal{L}(\mathcal{S})
$$

where $\mathcal{L}$ could also depend on other fields. To vary $I$ one first varies $\mathcal{S}$ as if it would be an unconstrained field with an unconstrained variation, finding an expression of the form

$$
\delta_{\mathcal{S}} I=\int \mathrm{d} t n e^{-\Phi} \operatorname{tr}\left(\delta \mathcal{S} F_{\mathcal{S}}\right)
$$

The equation of motion is not the vanishing of $F_{S}$ because $\delta \mathcal{S}$ is a constrained variation: since $\mathcal{S}^{2}=\mathbf{1}$ the variation $\delta \mathcal{S}$ satisfies $\delta \mathcal{S}=-\mathcal{S} \delta \mathcal{S S}$. We can write $\delta S$ in terms of an unconstrained variation $\delta K$ as follows:

$$
\delta \mathcal{S}=\frac{1}{2}(\delta K-\mathcal{S} \delta K \mathcal{S})
$$

Note that $\delta K$ cannot be written in terms of $\delta \mathcal{S}$; it has extra information that drops out in the combination shown in the above right-hand side. Substituting $\delta \mathcal{S}$ into the above variation $\delta_{\mathcal{S}} I$ we now get

$$
\delta_{\mathcal{S}} I=\int \mathrm{d} t n e^{-\Phi} \operatorname{tr}\left(\delta K E_{\mathcal{S}}\right), \quad E_{\mathcal{S}}=\frac{1}{2}\left(F_{\mathcal{S}}-\mathcal{S} F_{\mathcal{S}} \mathcal{S}\right) .
$$

The equation of motion is indeed $E_{\mathcal{S}}=0$, since $\delta K$ is an unconstrained variation. Note that any $E_{\mathcal{S}}$ defining an equation of motion for $\mathcal{S}$ satisfies

$$
E_{\mathcal{S}}=-\mathcal{S} E_{\mathcal{S}} \mathcal{S}
$$

There is finally an alternative rewriting of $\delta_{\mathcal{S}} I$ that can now be seen to be valid too:

$$
\delta_{\mathcal{S}} I=\int \mathrm{d} t n e^{-\Phi} \operatorname{tr}\left(\delta \mathcal{S} E_{\mathcal{S}}\right) .
$$

Using the expression for $E_{\mathcal{S}}$ in terms of $F_{S}$ and the constraint $\delta \mathcal{S}=-\mathcal{S} \delta \mathcal{S S}$ one quickly verifies that $\operatorname{tr}\left(\delta \mathcal{S} E_{\mathcal{S}}\right)=$ $\operatorname{tr}\left(\delta \mathcal{S} F_{\mathcal{S}}\right)$, and comparing with (2.12) we conclude that the above right-hand side is indeed equal to $\delta_{\mathcal{S}} I$. However, (2.16) is not the operational way to derive $E_{\mathcal{S}}$ by variation.

Applying the above discussion for the case of the action $I_{0}$ we now find by direct variation

$$
\begin{aligned}
\delta_{\mathcal{S}} I_{0} & =\int \mathrm{d} t n e^{-\Phi} \operatorname{tr}\left(\delta \mathcal{S} F_{\mathcal{S}}\right), \\
\text { with } \quad F_{\mathcal{S}} & \equiv \frac{1}{4}\left(\mathcal{D}^{2} \mathcal{S}-\mathcal{D} \Phi \mathcal{D S}\right)
\end{aligned}
$$

The equation of motion is now $E_{\mathcal{S}}=0$ with $E_{\mathcal{S}}=$ $\frac{1}{2}\left(F_{\mathcal{S}}-\mathcal{S} F_{\mathcal{S}} \mathcal{S}\right)$. It is straightforward to simplify the resulting expression for $E_{\mathcal{S}}$. For this we note from $\mathcal{S}=\mathbf{1}$ that $\mathcal{S}$ and $\mathcal{D S}$ anticommute:

$$
\mathcal{S D S}=-(\mathcal{D S}) \mathcal{S}
$$

Taking a further derivative of this equation we also find that

$$
\mathcal{S}\left(\mathcal{D}^{2} \mathcal{S}\right) \mathcal{S}=-\mathcal{D}^{2} \mathcal{S}-2 \mathcal{S}(\mathcal{D S})^{2}
$$

It is now a simple matter to show that

$$
E_{\mathcal{S}} \equiv \frac{1}{4}\left(\mathcal{D}^{2} \mathcal{S}+\mathcal{S}(\mathcal{D S})^{2}-(\mathcal{D} \Phi)(\mathcal{D S})\right)
$$

The lapse equation of motion follows by varying with respect to $n$. The variation is written in the form

$$
\delta_{n} I_{0}=\int \mathrm{d} t n e^{-\Phi} \frac{\delta n}{n} E_{n} .
$$

This is sensible, because one can readily show that a variation $\delta n$ transforms as a density and, as a result, the ratio $\delta n / n$ transforms as a scalar. The quantity $E_{n}$ is then a scalar as well. To compute this variation it is simplest to write the action $I_{0}$ in terms of ordinary time derivatives using (2.3):

$$
I_{0}=\int \mathrm{d} t e^{-\Phi} \frac{1}{n}\left(-\dot{\Phi}^{2}-\frac{1}{8} \operatorname{tr}\left(\dot{\mathcal{S}}^{2}\right)\right) .
$$

It follows that

$$
\delta_{n} I_{0}=\int \mathrm{d} t n e^{-\Phi}\left(\frac{\delta n}{n}\right)\left((\mathcal{D} \Phi)^{2}+\frac{1}{8} \operatorname{tr}\left((\mathcal{D S})^{2}\right)\right) .
$$

Comparing with (2.21) we have therefore found that

$$
E_{n} \equiv(\mathcal{D} \Phi)^{2}+\frac{1}{8} \operatorname{tr}\left((\mathcal{D S})^{2}\right)
$$

\section{B. $\boldsymbol{O}(\boldsymbol{d}, \boldsymbol{d})$ invariant $\boldsymbol{\alpha}^{\prime}$ corrections}

We will discuss a recursive procedure to write the duality invariant action in a canonical form. In this we use the 
two-derivative part of the action to do field redefinitions that allow us to simplify the higher-derivative parts of the action. The four-derivative part of the action is of order $\alpha^{\prime}$. The terms of order $\alpha^{\prime k}$ will have $2 k+2$ derivatives. We call $I_{k}$ the part of the action of order $\alpha^{\prime k}$, and it is a sum of terms,

$$
I_{k}=\sum_{p=1}^{N(k)} I_{k, p}
$$

where each term $I_{k, p}$ is a product of dilaton derivatives and traces of strings of $\mathcal{S}$ and its derivatives, in order to be duality invariant. There are only a finite number of terms $N(k)$ that can be constructed with the requisite number of derivatives. We have

$$
\begin{aligned}
I_{k, p}= & \alpha^{\prime k} c_{k, p} \int \mathrm{d} t n e^{-\Phi} \prod_{i}\left(\mathcal{D}^{j_{i}} \Phi\right)^{n_{i}} \\
& \times \prod_{l} \operatorname{tr}\left(\left(\mathcal{D}^{k_{l}^{1}} \mathcal{S}\right)^{m_{l}^{1}} \cdots\left(\mathcal{D}^{k_{l}^{q_{l}}} \mathcal{S}\right)^{m_{l}^{q_{l}}}\right),
\end{aligned}
$$

where $c_{k, p}$ are unit-free constants. For each $i$ in the first product, the values of the indices $j_{i}$ and $n_{i}$ denote the number of time derivatives and the power of the term. Because of the dilaton theorem [35], all nonderivative dependence on the dilaton is in the exponential prefactor, and we thus take $j_{i} \geq 1$. For each $l$ in the second product we get a trace factor. Inside the trace there are $q_{l}$ factors, each one a fixed power of a multiple time derivative of $\mathcal{S}$. This includes the possibility of some $k_{l}=0$ which means no derivatives of $\mathcal{S}$. This must be accompanied by $m_{l}=1$ since $\mathcal{S}^{2}=1$. Implicit above is a sum rule that requires that the total number of derivatives is $2 k+2$.

We will use a notation that makes clear what are the arguments of a function $X$ of fields. When we write

$$
X(A, B, \ldots)
$$

we mean that $X$ is strictly a function constructed from $A, B, \ldots$, without including time derivatives $\mathcal{D}$ of these functions. When we write

$$
X(\{A\},\{B\}, \ldots),
$$

with braces around the arguments, we mean $X$ is a function of $A, \mathcal{D} A, \mathcal{D}^{2} A, \ldots$, as well as $B, \mathcal{D} B, \mathcal{D}^{2} B, \ldots$. Mixed types of arguments are also possible: $X(A,\{B\})$ would depend only on $A$, but possibly on $B, \mathcal{D} B, \mathcal{D}^{2} B, \ldots$ In this notation equation (2.26) would be described as saying that

$$
I_{k, p}=\alpha^{\prime k} \int \mathrm{d} t n e^{-\Phi} X(\{\mathcal{D} \Phi\},\{\mathcal{S}\})
$$

for some choice of $X$. form

Our goal is now to prove that $I_{(k)}$ can be brought to the

$$
I_{k}=\alpha^{\prime k} \int \mathrm{d} t n e^{-\Phi} X(\mathcal{D S}) .
$$

This means that all we can have is a product of traces of powers of $\mathcal{D S}$. The proof will proceed inductively, assuming that all terms up to $I_{(k-1)}$ are of this form and then using field redefinitions to show that the action $I_{(k)}$ can be brought to this form (at the cost of changing the actions $I_{k+1}, I_{k+2}$, etc., which will be taken care of in the next induction step).

To this end we use various simple theorems established in [28]:

$$
\begin{gathered}
\operatorname{tr}(\mathcal{S})=\operatorname{tr}(\mathcal{D S})=\operatorname{tr}\left(\mathcal{D}^{2} \mathcal{S}\right)=\cdots=0, \\
\operatorname{tr}\left((\mathcal{D S})^{2 k+1}\right)=0, \quad \text { for } \quad k=0,1, \ldots, \\
\operatorname{tr}\left(\mathcal{S}(\mathcal{D S})^{k}\right)=0, \quad \text { for } \quad k=0,1, \ldots
\end{gathered}
$$

The strategy will be to work at each order of $\alpha^{\prime}$. Assume that we have succeeded in casting the action in the expected form (2.30) for $I_{2}, \ldots, I_{k-1}$. Then we are faced with bringing $I_{k}$ to the desired form. The action can be written as

$I=I_{0}+\sum_{p=1}^{k-1} I_{p}+\alpha^{\prime k} \int \mathrm{d} t n e^{-\Phi} X(\{\mathcal{D} \phi\},\{\mathcal{S}\})+\mathcal{O}\left(\alpha^{\prime k+1}\right)$.

We will then consider field redefinitions of the form

$$
\Phi \rightarrow \Phi+\alpha^{\prime k} \delta \Phi, \quad \mathcal{S} \rightarrow \mathcal{S}+\alpha^{k} \delta \mathcal{S} .
$$

To see how $I$ changes, we need only vary $I_{0}$, because this will generate $\mathcal{O}\left(\alpha^{\prime k}\right)$ terms that we are interested in. The variations of $I_{1}, \ldots, I_{k-1}$ generate terms of higher order than $\alpha^{\prime k}$ that are not relevant, but are of the prescribed form (2.26). The variations of $I_{0}$ are determined by the field equations and take the form

$$
\begin{aligned}
\delta I_{0} & =\alpha^{\prime k} \int \mathrm{d} t n e^{-\Phi}\left(\delta \Phi E_{\Phi}+\operatorname{tr}\left(\delta K E_{\mathcal{S}}\right)\right) \quad \text { with } \\
\delta \mathcal{S} & =\frac{1}{2}(\delta K-\mathcal{S} \delta K \mathcal{S}) .
\end{aligned}
$$

At each step we use these field redefinitions to remove undesirable terms from the $\mathcal{O}\left(\alpha^{\prime k}\right)$ part of the action.

We elaborate and systematize some of the derivations of [28], carefully proving several statements that lead to the desired claim. 
(1) A factor $\mathcal{D}^{2} \Phi$ in an action can be replaced by a factor of $Q_{\Phi}$ with only first derivatives.

Consider a generic such term $Z_{k}$ at order $\alpha^{\prime k}$ as part of (2.34):

$$
Z_{k}=\alpha^{\prime k} \int \mathrm{d} t n e^{-\Phi} X(\{\mathcal{D} \Phi\},\{\mathcal{S}\}) \mathcal{D}^{2} \Phi .
$$

With a dilaton field redefinition equation (2.36) gives us

$$
\begin{aligned}
\delta I_{0}= & \alpha^{\prime k} \int \mathrm{d} t n e^{-\Phi} 2 \delta \Phi \\
& \times\left(\mathcal{D}^{2} \Phi-\frac{1}{2}(\mathcal{D} \Phi)^{2}+\frac{1}{16} \operatorname{tr}\left((\mathcal{D} S)^{2}\right)\right) .
\end{aligned}
$$

If we choose

$$
2 \delta \Phi=-X(\{\mathcal{D} \Phi\},\{\mathcal{S}\}),
$$

the shift in $I_{0}$ cancels $Z_{k}$ completely and replaces it with

$$
\begin{aligned}
Z_{k}^{\prime}= & \alpha^{\prime k} \int \mathrm{d} \text { tne } e^{-\Phi} X(\{\mathcal{D} \Phi\},\{\mathcal{S}\}) \\
& \times\left(\frac{1}{2}(\mathcal{D} \Phi)^{2}-\frac{1}{16} \operatorname{tr}\left((\mathcal{D S})^{2}\right)\right)
\end{aligned}
$$

The net effect is that the following replacement is allowed in the $\mathcal{O}\left(\alpha^{\prime k}\right)$ action:

$$
\mathcal{D}^{2} \Phi \rightarrow Q_{\Phi} \equiv \frac{1}{2}(\mathcal{D} \Phi)^{2}-\frac{1}{16} \operatorname{tr}\left((\mathcal{D} S)^{2}\right),
$$

with $Q_{\Phi}$ having only first derivatives.

(2) A factor of $\mathcal{D}^{2} \mathcal{S}$ in an action can be replaced by a factor of $Q_{\mathcal{S}}$ with only first-order derivatives.

Consider a generic term $Z_{k}$ at order $\alpha^{\prime k}$ with a second derivative on $\mathcal{S}$ :

$Z_{k}=\alpha^{\prime k} \int \mathrm{d} t n e^{-\Phi} X(\{\mathcal{D} \Phi\},\{\mathcal{S}\}) \operatorname{tr}\left(\mathcal{G} \mathcal{D}^{2} \mathcal{S}\right)$,

where $\mathcal{G}$ is a matrix of type $\mathcal{G}(\{\mathcal{S}\})$. With a field redefinition of $\mathcal{S}$, Eq. (2.36) gives

$$
\begin{aligned}
\delta I_{0}= & \alpha^{\prime k} \int \mathrm{d} t n e^{-\Phi} \operatorname{tr}\left[\frac { 1 } { 4 } \delta K \left(\mathcal{D}^{2} \mathcal{S}+\mathcal{S}(\mathcal{D S})^{2}\right.\right. \\
& -(\mathcal{D} \Phi)(\mathcal{D} S))]
\end{aligned}
$$

Choosing the matrix $\delta K$ [which determines $\delta \mathcal{S}=$ $\left.\frac{1}{2}(\delta K-\mathcal{S} \delta K \mathcal{S})\right]$ to be

$$
\frac{1}{4} \delta K=-X(\{\mathcal{D} \Phi\},\{\mathcal{S}\}) \mathcal{G}
$$

and realizing that $X$ is a scalar that can go out of the trace, it gives

$$
\begin{aligned}
\delta I_{0}= & -\alpha^{\prime k} \int \mathrm{d} t n e^{-\Phi} X(\{\mathcal{D} \Phi\},\{\mathcal{S}\}) \\
& \times \operatorname{tr}\left[\mathcal{G}\left(\mathcal{D}^{2} \mathcal{S}+\mathcal{S}(\mathcal{D S})^{2}-(\mathcal{D} \Phi)(\mathcal{D} S)\right)\right]
\end{aligned}
$$

This variation cancels the $Z_{k}$ term above and replaces it with

$$
\begin{aligned}
Z_{k}^{\prime}= & \alpha^{\prime k} \int \mathrm{d} t n e^{-\Phi} X(\{\mathcal{D} \Phi\},\{\mathcal{S}\}) \\
& \times \operatorname{tr}\left(\mathcal{G}\left(-\mathcal{S}(\mathcal{D S})^{2}+(\mathcal{D} \Phi)(\mathcal{D} S)\right)\right) .
\end{aligned}
$$

The net effect is that the following replacement is allowed in the $\mathcal{O}\left(\alpha^{\prime k}\right)$ action:

$$
\mathcal{D}^{2} \mathcal{S} \rightarrow Q_{\mathcal{S}} \equiv-\mathcal{S}(\mathcal{D S})^{2}+\mathcal{D} \Phi \mathcal{D} \mathcal{S},
$$

with $Q_{\mathcal{S}}$ having at most first derivatives.

(3) Any action can be reduced so that it only has first time derivatives of $\Phi$.

We already know how to eliminate terms with two derivatives on the dilaton. Suppose we have an action with more than two derivatives of $\Phi$. This can always be written in the form

$$
\begin{aligned}
Z_{k} & =\alpha^{\prime k} \int \mathrm{d} t n e^{-\Phi} X(\{\mathcal{D} \Phi\},\{\mathcal{S}\}) \mathcal{D}^{p+2} \Phi, \\
& <<p \leq 2 k
\end{aligned}
$$

where the dilaton factor to the right has more than two derivatives. Now, write $\mathcal{D}^{p+2} \Phi=\mathcal{D}^{p}\left(\mathcal{D}^{2} \Phi\right)$ and integrate by parts the $\mathcal{D}^{p}$, finding

$$
Z_{k}=\alpha^{\prime k} \int \mathrm{d} \operatorname{tn}(-\mathcal{D})^{p}\left(e^{-\Phi} X\right) \mathcal{D}^{2} \Phi,
$$

suppressing the arguments of $X$. We can now use property 1 to eliminate the $\mathcal{D}^{2} \Phi$, replacing $Z_{k}$ by

$$
\begin{aligned}
Z_{k}^{\prime}= & \alpha^{\prime k} \int \mathrm{d} \operatorname{tn}(-\mathcal{D})^{p}\left(e^{-\Phi} X\right) \\
& \times\left(\frac{1}{2}(\mathcal{D} \Phi)^{2}-\frac{1}{16} \operatorname{tr}(\mathcal{D S})^{2}\right)
\end{aligned}
$$

Now we integrate back the $p$ derivatives of the left factor, one by one. At each step this will generate a second-order time derivative of the second factor, but these can be reduced to first derivatives by properties 1 and 2 . We can write this formally 
defining a derivative operator $\overline{\mathcal{D}}$ that acts on functions $F$ of $\mathcal{S}, \mathcal{D S}$, and $\mathcal{D} \Phi$ :

$$
\begin{aligned}
& (\overline{\mathcal{D}} F)(\mathcal{S}, \mathcal{D} \mathcal{S}, \mathcal{D} \Phi) \\
& \left.\quad \equiv \mathcal{D}(F(\mathcal{S}, \mathcal{D} \mathcal{S}, \mathcal{D} \Phi))\right|_{\mathcal{D}^{2} \Phi \rightarrow Q_{\Phi}, \mathcal{D}^{2} \mathcal{S} \rightarrow Q_{\mathcal{S}}} .
\end{aligned}
$$

The replacements here are those in (2.41) and (2.47). Note that after taking a $\overline{\mathcal{D}}$ derivative, the result is still a function of only $\mathcal{S}, \mathcal{D S}$, and $\mathcal{D} \Phi$. It follows now that $Z_{k}$ in (2.48) is eventually replaced by

$$
Z_{k}^{\prime \prime}=\alpha^{\prime k} \int \mathrm{d} t n e^{-\Phi} X(\{\mathcal{D} \Phi\},\{\mathcal{S}\}) \overline{\mathcal{D}}^{p} Q_{\Phi}
$$

The factor to the right of $X$ contains only first derivatives of $\Phi$ and at most first derivatives of $\mathcal{S}$. Because of (2.33), the factor to the right of $X$ cannot contain $\mathcal{S}$; it must be built explicitly with $\mathcal{D S}$ only.

Should $X$ above still contain factors with more than two derivatives of $\Phi$ the procedure is carried through again for each factor. Proceeding in this way we can reduce the higher derivative factors in $\Phi$ one by one to first order in time derivatives. This step has shown that the most general form of the action is

$$
I_{k}=\alpha^{\prime k} \int \mathrm{d} t n e^{-\Phi} X(\mathcal{D} \Phi,\{\mathcal{S}\}) .
$$

This means that, apart from the $e^{-\Phi}$ factor, only first derivatives of the dilaton appear.

(4) Any action can be reduced so that it only has first time derivatives of $\mathcal{S}$.

Assume there is a trace factor that has a time derivative of $\mathcal{S}$ of a degree higher than 2. Using the form of the action (2.53) we already know to be true, we write the action separating out such a term:

$$
\begin{aligned}
Z_{k} & =\alpha^{\prime k} \int \mathrm{d} t n e^{-\Phi} X(\mathcal{D} \Phi,\{\mathcal{S}\}) \operatorname{tr}\left[\mathcal{G}(\{\mathcal{S}\}) \mathcal{D}^{p+2} \mathcal{S}\right] \\
0 & <p \leq 2 k
\end{aligned}
$$

Clearly $X$ must contain $2 k-p$ derivatives, but we need not include this in the notation. Moreover, $\mathcal{G}$ is a matrix built as products of $\mathcal{S}$ and its derivatives. Defining the matrix $\mathcal{F}$ as follows:

$$
\mathcal{F}(\mathcal{D} \Phi,\{\mathcal{S}\}) \equiv e^{-\Phi} X(\mathcal{D} \Phi,\{\mathcal{S}\}) \mathcal{G}(\{\mathcal{S}\}),
$$

the action above takes the form

$$
Z_{k}=\alpha^{\prime k} \int \mathrm{d} \operatorname{tn} \operatorname{tr}\left[\mathcal{F} \mathcal{D}^{p+2} \mathcal{S}\right] .
$$

For brevity, we do not indicate the arguments of $\mathcal{F}$, which are given in (2.55). In this form integration by parts is straightforward and folds the derivatives into
$\mathcal{F}$. We integrate by parts $\mathcal{D}^{p}$ and then are allowed to replace $\mathcal{D}^{2} \mathcal{S}$ by $Q_{\mathcal{S}}$, finding that $Z_{k}$ is replaced by

$$
Z_{k}^{\prime}=\alpha^{\prime k} \int \mathrm{d} t n \operatorname{tr}\left[(-\mathcal{D})^{p} \mathcal{F} Q_{\mathcal{S}}\right]
$$

The derivatives are then folded back into $Q_{\mathcal{S}}$, one at a time, at each step eliminating the second derivatives that are generated. As explained in property 3, each $(-\mathcal{D})$ derivative becomes a $\overline{\mathcal{D}}$ derivative, and we find that $Z_{k}^{\prime}$ becomes

$$
Z_{k}^{\prime}=\alpha^{\prime k} \int \mathrm{d} t n \operatorname{tr}\left[\mathcal{F} \overline{\mathcal{D}}^{p} Q_{\mathcal{S}}\right]
$$

Using the definition of $\mathcal{F}$ we now note that (2.54) has become

$$
Z_{k}^{\prime \prime}=\alpha^{\prime k} \int \mathrm{d} t n e^{-\Phi} X(\mathcal{D} \Phi,\{\mathcal{S}\}) \operatorname{tr}\left[\mathcal{G}(\{\mathcal{S}\}) \overline{\mathcal{D}}^{p} Q_{\mathcal{S}}\right],
$$

achieving the desired elimination of the higher derivative of $\mathcal{S}$ in terms of first derivatives of $\Phi$ and up to first derivatives of $\mathcal{S}$. This procedure can be iterated until all higher derivatives of $\mathcal{S}$ are eliminated. At his point only $\mathcal{S}$ and $\mathcal{D S}$ can appear and, as explained before, this means that only $\mathcal{D} \mathcal{S}$ can appear. We have thus shown that terms of $I_{k}$, already put in the form (2.53), can be simplified further to be set in the form

$$
I_{k}=\alpha^{\prime k} \int \mathrm{d} t n e^{-\Phi} X(\mathcal{D} \Phi, \mathcal{D S}) .
$$

Each term $I_{k, p}$ in such an action is written in the form

$$
I_{k, p}=\alpha^{\prime k} \int \mathrm{d} t n e^{-\Phi}(\mathcal{D} \Phi)^{p} X(\mathcal{D S}) .
$$

We will now show that, in fact, any dilaton derivative or power of a dilaton derivative can be eliminated.

(5) Any action $I_{k}$, with $k>1$ is equivalent to one without any appearance of $\mathcal{D} \Phi$.

Suppose we have an action $I_{k}$ with a term

$$
I_{k, p}=\int \mathrm{d} t n e^{-\Phi}(\mathcal{D} \Phi)^{p} X_{l}(\mathcal{D S}) .
$$

Here $X_{l}$ is an arbitrary (generally multitrace) invariant:

$$
\begin{aligned}
X_{l}(\mathcal{D S}) & =\operatorname{tr}\left[(\mathcal{D S})^{l_{1}}\right] \cdots \operatorname{tr}\left[(\mathcal{D S})^{l_{n}}\right], \\
l & =l_{1}+\cdots+l_{n} .
\end{aligned}
$$


In a moment we will need that the time derivative of this function has a simple form. As we take a derivative we generate $\mathcal{D}^{2} \mathcal{S}$ factors within the traces. Each such factor can be replaced by $Q_{\mathcal{S}}=-\mathcal{S}(\mathcal{D S})^{2}+\mathcal{D} \Phi \mathcal{D} \mathcal{S}$, but since traces with a single $\mathcal{S}$ multiplying $\mathcal{D S}$ factors vanish [Eq. (2.33)], the replacement is effectively $\mathcal{D}^{2} \mathcal{S} \rightarrow \mathcal{D} \Phi \mathcal{D S}$. As a result, inside an integral representing a term in $I_{k}$, the following replacement is allowed:

$$
\mathcal{D} X_{l} \sim l \mathcal{D} \Phi X_{l}(\mathcal{D S})
$$

We begin by rewriting (2.62) with one factor of $\mathcal{D} \Phi$ written as a derivative of $e^{-\Phi}$, and then proceed to do integration by parts:

$$
\begin{aligned}
I_{k, p}= & -\int \mathrm{d} t n \mathcal{D}\left(e^{-\Phi}\right)(\mathcal{D} \Phi)^{p-1} X_{l} \\
= & \int \mathrm{d} t n e^{-\Phi}\left((p-1)(\mathcal{D} \Phi)^{p-2} \mathcal{D}^{2} \Phi X_{l}\right. \\
& \left.+(\mathcal{D} \Phi)^{p-1} \mathcal{D} X_{l}\right) .
\end{aligned}
$$

Replacing $\mathcal{D}^{2} \Phi$ by $Q_{\Phi}$ and using the above evaluation of $\mathcal{D} X_{l}$ we get

$$
\begin{aligned}
I_{k, p} \sim & \int \mathrm{d} t n e^{-\Phi}\left((p-1)(\mathcal{D} \Phi)^{p-2}\right. \\
& \left.\times\left(\frac{1}{2}(\mathcal{D} \Phi)^{2}-\frac{1}{16} \operatorname{tr}(\mathcal{D S})^{2}\right) X_{l}+l(\mathcal{D} \Phi)^{p} X_{l}\right) \\
= & \int \mathrm{d} t n e^{-\Phi}\left(\frac{1}{2}(p+2 l-1)(\mathcal{D} \Phi)^{p} X_{l}\right. \\
& \left.-\frac{1}{16}(p-1)(\mathcal{D} \Phi)^{p-2} \operatorname{tr}(\mathcal{D S})^{2} X_{l}\right)
\end{aligned}
$$

The first term is of the same form as the original (2.62), and thus bringing it to the left-hand side we have the equivalence

$$
\begin{aligned}
\frac{1}{2}(3-p-2 l) \int \mathrm{d} t n e^{-\Phi}(\mathcal{D} \Phi)^{p} X_{l} \\
\quad=-\frac{1}{16}(p-1) \int \mathrm{d} t n e^{-\Phi}(\mathcal{D} \Phi)^{p-2} \operatorname{tr}(\mathcal{D S})^{2} X_{l},
\end{aligned}
$$

and thus in actions we can replace

$$
\begin{aligned}
& \int \mathrm{d} t n e^{-\Phi}(\mathcal{D} \Phi)^{p} X_{l}(\mathcal{D S}) \\
& \sim \frac{1}{8}\left(\frac{p-1}{p+2 l-3}\right) \int \mathrm{d} t n e^{-\Phi}(\mathcal{D} \Phi)^{p-2} \\
& \quad \times \operatorname{tr}(\mathcal{D S})^{2} X_{l}(\mathcal{D S}) .
\end{aligned}
$$

Let us note that the denominator on the right-hand side never vanishes for any case of interest. Since the total number of derivatives in the term is $p+l$, for a term in an action $I_{k}$, the denominator takes the value

$$
\begin{aligned}
(p+l)+l-3 & =2 k+2+l-3=2 k+l-1 \geq 1, \\
\text { for } k & \geq 1 .
\end{aligned}
$$

The formula can be used recursively to reduce the power of $\mathcal{D} \Phi$ in steps of two; in each step $p$ decreases by two units and $l$ increases by two units [since $\operatorname{tr}(\mathcal{D S})^{2} X_{l}$ is an $X_{l+2}$ object], and at no stage will the denominator vanish. If $p=2 m$ is even, it follows from (2.68) that we have

$$
\begin{aligned}
& \int \mathrm{d} t n e^{-\Phi}(\mathcal{D} \Phi)^{2 m} X_{l}(\mathcal{D S}) \\
& \sim C \int \mathrm{d} t n e^{-\Phi}\left[\operatorname{tr}(\mathcal{D S})^{2}\right]^{m} X_{l}(\mathcal{D S}),
\end{aligned}
$$

where $C$ is a constant that is easily determined. Note also that for $p=1$ Eq. (2.68) tells us that

$$
\int \mathrm{d} t n e^{-\Phi} \mathcal{D} \Phi X_{l}(\mathcal{D S}) \sim 0 .
$$

For the case $l=1$, where the prefactor in (2.68) gives zero divided by zero, the left-hand side vanishes too because $\operatorname{tr}(\mathcal{D S})=0$. Since the recursion relation works in steps of two units, the vanishing of terms with a single $\mathcal{D} \Phi$ implies that

$$
\int \mathrm{d} t n e^{-\Phi}(\mathcal{D} \Phi)^{2 m+1} X_{l}(\mathcal{D S}) \sim 0 .
$$

The formula (2.68) also applies when $l=0$ and $X_{l}=1$, in which case it gives

$$
\begin{aligned}
\int \mathrm{d} t n e^{-\Phi}(\mathcal{D} \Phi)^{p} \sim & \frac{1}{8}\left(\frac{p-1}{p-3}\right) \\
& \times \int \mathrm{d} t n e^{-\Phi}(\mathcal{D} \Phi)^{p-2} \operatorname{tr}(\mathcal{D S})^{2} .
\end{aligned}
$$

The denominator in the prefactor only vanishes for $p=3$ which is not relevant for any action $I_{k}$. The remarks above apply again. When $p=2 m$ is even, the term can be reduced to one containing no dilaton derivatives and just $\left[\operatorname{tr}(\mathcal{D S})^{2}\right]^{m}$. If $p$ is odd, the term vanishes because it does for $p=1$.

We have shown so far that any term $I_{k, p}$ in the action $I_{k}$ takes the form (2.61). Since we have now learned that powers of derivatives of the dilaton can be 
eliminated completely, we have shown that any term in $I_{k}$ takes the claimed form (2.30):

$$
I_{k}=\alpha^{\prime k} \int \mathrm{d} t n e^{-\Phi} X(\mathcal{D S}) .
$$

While this is a great simplification, further simplification can be achieved by using redefinitions of the lapse function $n(t)$. We explore this next.

\section{Lapse redefinitions}

We have confirmed that up to $O(d, d)$ covariant redefinitions of $\mathcal{S}$ and $\Phi$ one only needs to consider (arbitrary powers of) first time derivatives of $\mathcal{S}$ and no time derivatives of $\Phi$. Thus, the most general action takes the following form:

$I=\int \mathrm{d} t n e^{-\Phi}\left(L_{0}+\alpha^{\prime} L_{1}+\left(\alpha^{\prime}\right)^{2} L_{2}+\left(\alpha^{\prime}\right)^{3} L_{3}+\cdots\right)$,

where the general forms of $L_{1}, L_{2}$, and $L_{3}$ are

$$
\begin{aligned}
L_{1}= & a_{1} \operatorname{tr}(\mathcal{D S})^{4}+a_{2}\left[\operatorname{tr}(\mathcal{D S})^{2}\right]^{2}, \\
L_{2}= & b_{1} \operatorname{tr}(\mathcal{D S})^{6}+b_{2} \operatorname{tr}(\mathcal{D S})^{4} \operatorname{tr}(\mathcal{D S})^{2}+b_{3}\left[\operatorname{tr}(\mathcal{D S})^{2}\right]^{3}, \\
L_{3}= & c_{1} \operatorname{tr}(\mathcal{D S})^{8}+c_{2}\left[\operatorname{tr}(\mathcal{D S})^{4}\right]^{2}+c_{3} \operatorname{tr}(\mathcal{D S})^{6} \operatorname{tr}(\mathcal{D S})^{2} \\
& +c_{4} \operatorname{tr}(\mathcal{D S})^{4}\left[\operatorname{tr}(\mathcal{D S})^{2}\right]^{2}+c_{5}\left[\operatorname{tr}(\mathcal{D S})^{2}\right]^{4}
\end{aligned}
$$

We will now show that using lapse redefinitions, together with further dilaton redefinitions, we can remove any term in the action $I$ that has a $\operatorname{tr}(\mathcal{D S})^{2}$ factor. This leaves, as one can see, the first term in $L_{1}$, the first term in $L_{2}$, and the first two terms in $L_{3}$.

As before, we work at fixed orders of $\alpha^{\prime}$ recursively: at each fixed order $\alpha^{\prime k}$ we eliminate terms with a $\operatorname{tr}(\mathcal{D S})^{2}$ factor by doing a redefinition of $n$ of order $\alpha^{\prime k}$ that contributes through the variation of $I_{0}$. This variation was given in (2.23):

$\delta_{n} I_{0}=\int \mathrm{d} t n e^{-\Phi}\left(\frac{\delta n}{n}\right)\left((\mathcal{D} \Phi)^{2}+\frac{1}{8} \operatorname{tr}(\mathcal{D S})^{2}\right)$.

Consider now a general term in $I_{k, p}$ with a $\operatorname{tr}(\mathcal{D S})^{2}$ factor:

$$
I_{k, p}=\alpha^{\prime k} \int \mathrm{d} t n e^{-\Phi} X_{2 k}(\mathcal{D S}) \operatorname{tr}(\mathcal{D S})^{2} .
$$

Here $X_{2 k}(\mathcal{D S})$ denotes a term with $2 k$ derivatives built from products of traces of powers of $\mathcal{D S}$. It does not matter if $X_{2 k}$ has additional factors of $\operatorname{tr}(\mathcal{D S})^{2}$; it may. We will consider a redefinition of the form

$$
\frac{\delta n}{n}=\beta \alpha^{\prime k} X_{2 k}(\mathcal{D S})
$$

where $\beta$ is a constant to be determined. The associated variation of $I_{0}$ is therefore

$\delta_{n} I_{0}=\beta \alpha^{\prime k} \int \mathrm{d} t n e^{-\Phi} X_{2 k}(\mathcal{D S})\left((\mathcal{D} \Phi)^{2}+\frac{1}{8} \operatorname{tr}(\mathcal{D S})^{2}\right)$.

This counts as an additional term in the action. Note the second term in parentheses gives a contribution that could cancel the term in $I_{k, p}$ above. The first term also does, if we use identity (2.68) which amounts to a further dilaton redefinition. This identity with $p=2$ and $l=2 k$ allows us to write

$$
\begin{aligned}
\delta_{n} I_{0}= & \beta \alpha^{\prime k} \int \mathrm{d} t n e^{-\Phi} X_{2 k}(\mathcal{D S}) \\
& \times\left(\frac{1}{8(4 k-1)} \operatorname{tr}(\mathcal{D S})^{2}+\frac{1}{8} \operatorname{tr}(\mathcal{D S})^{2}\right) \\
= & \frac{\beta k}{2(4 k-1)} \alpha^{\prime k} \int \mathrm{d} t n e^{-\Phi} X_{2 k}(\mathcal{D S}) \operatorname{tr}(\mathcal{D S})^{2} .
\end{aligned}
$$

One can cancel $I_{k, p}$ in (2.78) by choosing $\beta$ such that $\frac{\beta k}{2(4 k-1)}=-1$. Since this can always be done, this completes our proof that any term with $\operatorname{ar}(\mathcal{D S})^{2}$ factor can be set to zero.

Given this result, the most general $\alpha^{\prime}$ corrections up to order $\left(\alpha^{\prime}\right)^{3}$ given in (2.76) can be simplified to take the form

$$
\begin{aligned}
& L_{1}=a_{1} \operatorname{tr}(\mathcal{D S})^{4}, \\
& L_{2}=b_{1} \operatorname{tr}(\mathcal{D S})^{6}, \\
& L_{3}=c_{1} \operatorname{tr}(\mathcal{D S})^{8}+c_{2}\left[\operatorname{tr}(\mathcal{D S})^{4}\right]^{2} .
\end{aligned}
$$

Thus, refining the classification of [28], we find that the number of independent coefficients in the $\mathcal{O}\left(\left(\alpha^{\prime}\right)^{k}\right)$ action is given by the number of partitions of $k+1$ that do not use 1 , which in the literature is sometimes denoted by $p(k+1,2)$. Using Euler's generating function for the unrestricted number $p(n)$ of partitions of the integer $n$, it is easy to prove that this equals

$$
p(k+1,2)=p(k+1)-p(k) ;
$$

see, e.g., [36]. This proves our statement in the Introduction.

\section{EQUATIONS OF MOTION AND NOETHER CHARGES}

In this section we determine the explicit equations of motion for the special case that only single trace invariants are included, which is, however, sufficient for the cosmological applications in the next section. Moreover, we discuss Bianchi identities and the conserved Noether charges corresponding to $O(d, d)$ duality invariance. 


\section{A. Equations of motion}

We now compute the equations of motion obtained from the action (2.3) upon including single trace higher derivative terms. Upon setting $n=1$ this action reads

$$
I \equiv \int \mathrm{d} t e^{-\Phi}\left(-\dot{\Phi}^{2}+\sum_{k=1}^{\infty} \alpha^{\prime k-1} c_{k} \operatorname{tr}\left(\dot{\mathcal{S}}^{2 k}\right)\right),
$$

where we included the lowest-order term in the sum, with $c_{1}=-\frac{1}{8}$. The higher $c_{k}$ coefficients are, of course, only partially known (e.g., $c_{2}=\frac{1}{64}$ for bosonic string theory, $c_{2}=\frac{1}{128}$ for heterotic string theory, and $c_{2}=0$ for type II string theories).

In order to derive the equation of motion for $\mathcal{S}$ we follow the steps reviewed in Sec. II A. The variation with respect to $\mathcal{S}$ of (3.1) reads

$$
\begin{aligned}
\delta_{\mathcal{S}} I & =\sum_{k=1}^{\infty} \alpha^{\prime k-1} c_{k} \int \mathrm{d} t e^{-\Phi}(2 k) \operatorname{tr}\left(\frac{d \delta \mathcal{S}}{d t} \dot{\mathcal{S}}^{2 k-1}\right) \\
& =\sum_{k=1}^{\infty} \alpha^{\prime k-1} c_{k} \int \mathrm{d} t e^{-\Phi}(2 k) \operatorname{tr}\left(\frac{d}{d t}\left[\delta \mathcal{S} \dot{\mathcal{S}}^{2 k-1}\right]-\left[\delta \mathcal{S} \frac{d}{d t} \dot{\mathcal{S}}^{2 k-1}\right]\right) \\
& =\sum_{k=1}^{\infty} \alpha^{\prime k-1} c_{k} \int \mathrm{d} t e^{-\Phi} \operatorname{tr}\left(\delta \mathcal{S}(2 k)\left[\dot{\Phi} \dot{\mathcal{S}}^{2 k-1}-\frac{d}{d t} \dot{\mathcal{S}}^{2 k-1}\right]\right) \\
& =\int \mathrm{d} t e^{-\Phi} \operatorname{tr}\left(\delta \mathcal{S} \sum_{k=1}^{\infty} \alpha^{\prime k-1} c_{k}(2 k)\left[\dot{\Phi}^{2 k-1}-\frac{d}{d t} \dot{\mathcal{S}}^{2 k-1}\right]\right),
\end{aligned}
$$

where we discarded total time derivatives. The above defines $F_{\mathcal{S}}$, and therefore $E_{\mathcal{S}}$ is given by

$$
\begin{aligned}
E_{\mathcal{S}}= & \frac{1}{2} \sum_{k=1}^{\infty} \alpha^{k-1} c_{k}(2 k)\left(\left[\dot{\Phi} \dot{\mathcal{S}}^{2 k-1}-\frac{d}{d t} \dot{\mathcal{S}}^{2 k-1}\right]\right. \\
& \left.-\mathcal{S}\left[\dot{\Phi} \dot{\mathcal{S}}^{2 k-1}-\frac{d}{d t} \dot{\mathcal{S}}^{2 k-1}\right] \mathcal{S}\right) .
\end{aligned}
$$

This form can be simplified by using the anticommutativity of $\mathcal{S}$ and $\dot{\mathcal{S}}$ :

$E_{\mathcal{S}}=\sum_{k=1}^{\infty} \alpha^{\prime k-1} c_{k} k\left(2 \dot{\Phi} \dot{\mathcal{S}}^{2 k-1}-\frac{d}{d t} \dot{\mathcal{S}}^{2 k-1}+\mathcal{S} \frac{d}{d t} \dot{\mathcal{S}}^{2 k-1} \mathcal{S}\right)$.

This equation can be further simplified as follows: using $\mathcal{S} \ddot{\mathcal{S}}=-\ddot{\mathcal{S}} \mathcal{S}-2 \dot{\mathcal{S}}^{2}$, which follows by taking the second derivative of $\mathcal{S}^{2}=\mathbf{1}$, it is straightforward to verify

$$
\mathcal{S} \frac{d}{d t} \dot{\mathcal{S}}^{2 k-1} \mathcal{S}=-\frac{d}{d t} \dot{\mathcal{S}}^{2 k-1}-2 \mathcal{S} \dot{\mathcal{S}}^{2 k}
$$

Using this in (3.4), $E_{\mathcal{S}}$ finally reduces to

$E_{\mathcal{S}}=-2 \sum_{k=1}^{\infty} \alpha^{\prime k-1} c_{k} k\left(\frac{d}{d t} \dot{\mathcal{S}}^{2 k-1}-\dot{\Phi}^{2 k-1}+\mathcal{S}^{2 k}\right)$

The equation of motion for $\mathcal{S}$ is $E_{\mathcal{S}}=0$.

In order to find the equation of motion for $n$ we restore this dependence in the action (3.1):

$$
\begin{aligned}
I & \equiv \int \mathrm{d} t n e^{-\Phi}\left(-(\mathcal{D} \Phi)^{2}+\sum_{k=1}^{\infty} \alpha^{\prime k-1} c_{k} \operatorname{tr}(\mathcal{D S})^{2 k}\right) \\
& =\int \mathrm{d} t e^{-\Phi}\left(-\frac{1}{n} \dot{\Phi}^{2}+\sum_{k=1}^{\infty} \frac{\alpha^{\prime k-1}}{n^{2 k-1}} c_{k} \operatorname{tr}\left(\dot{\mathcal{S}}^{2 k}\right)\right) .
\end{aligned}
$$

One quickly finds by variation of $n$ and then back to covariant notation that

$$
I \equiv \int \mathrm{d} t n e^{-\Phi} \frac{\delta n}{n}\left((\mathcal{D} \Phi)^{2}-\sum_{k=1}^{\infty} \alpha^{\prime k-1}(2 k-1) c_{k} \operatorname{tr}\left((\mathcal{D S})^{2 k}\right)\right)
$$

The object in between big parentheses is $E_{n}$. It is straightforward to compute the $\Phi$ variation of (3.1). To recapitulate let us record the general variation. Recall that $E_{\mathcal{S}}$ can be used directly in the variation [see (2.16)], and we can set $n=1$ in the $E_{n}$ equation, too. We have

$$
\delta I=\int \mathrm{d} t n e^{-\Phi}\left(\delta \Phi E_{\Phi}+\operatorname{tr}\left(\delta \mathcal{S} E_{\mathcal{S}}\right)+\frac{\delta n}{n} E_{n}\right),
$$

with the (off-shell) functions

$$
\begin{aligned}
& E_{\Phi} \equiv 2 \ddot{\Phi}-\dot{\Phi}^{2}-\sum_{k=1}^{\infty} \alpha^{\prime k-1} c_{k} \operatorname{tr}\left(\dot{\mathcal{S}}^{2 k}\right) \\
& E_{\mathcal{S}} \equiv-2 \sum_{k=1}^{\infty} \alpha^{\prime k-1} k c_{k}\left(\frac{d}{d t} \dot{\mathcal{S}}^{2 k-1}-\dot{\Phi}^{2 k-1}+\mathcal{S} \dot{\mathcal{S}}^{2 k}\right) \\
& E_{n} \equiv \dot{\Phi}^{2}-\sum_{k=1}^{\infty} \alpha^{\prime k-1}(2 k-1) c_{k} \operatorname{tr}\left(\dot{\mathcal{S}}^{2 k}\right)
\end{aligned}
$$


These expressions satisfy a Bianchi identity as a consequence of time reparametrization invariance of $I$ under the field variations:

$$
\delta_{\xi} \mathcal{S}=\xi \dot{\mathcal{S}}, \quad \delta_{\xi} \Phi=\xi \dot{\Phi}, \quad \delta_{\xi} n=\partial_{t}(\xi n) .
$$

Specializing (3.9) to these variations and integrating by parts we then infer

$0=\delta_{\xi} I=\int \mathrm{d} t e^{-\Phi} \xi\left(\dot{\Phi} E_{\Phi}+\operatorname{tr}\left(\dot{\mathcal{S}} E_{\mathcal{S}}\right)-e^{\Phi} \partial_{t}\left(e^{-\Phi} E_{n}\right)\right)$,

where we set $n=1$, which is legal after variation. Since the integral vanishes for arbitrary $\xi$, we infer the

Bianchiidentity: $\dot{\Phi}\left(E_{\Phi}+E_{n}\right)+\operatorname{tr}\left(\dot{\mathcal{S}} E_{\mathcal{S}}\right)=\frac{d}{d t} E_{n}$.

This identity may also be verified by a direct computation using (3.10). It will be instrumental because it implies that it is sufficient to solve the equations

$$
E_{\Phi}+E_{n}=0, \quad E_{\mathcal{S}}=0,
$$

and to impose the lapse equation (or Hamiltonian constraint) $E_{n}=0$ only on initial data. The lapse equation will hold for all times as a consequence of (3.13). Note that

$$
E_{\Phi}+E_{n}=2 \ddot{\Phi}-\sum_{k=1}^{\infty} \alpha^{\prime k-1}(2 k) c_{k} \operatorname{tr}\left(\dot{\mathcal{S}}^{2 k}\right)
$$

\section{B. Noether charges}

We will now compute the Noether charges corresponding to $O(d, d)$ invariance. To this end we consider the infinitesimal $O(d, d)$ transformations that leave the action invariant and that are given by

$$
\delta_{\tau} \mathcal{S}=\tau \mathcal{S}-\mathcal{S} \tau, \quad \delta_{\tau} \Phi=0,
$$

where $\tau=\left(\tau^{M}{ }_{N}\right) \in \mathfrak{g} \mathfrak{o}(d, d)$, thus satisfying

$$
\tau \eta+\eta \tau^{t}=0
$$

In using matrix notation we also have $S=\left(S^{M}{ }_{N}\right)=\eta \mathcal{H}$ with $\eta=\left(\eta^{M N}\right)$ and $\mathcal{H}=\mathcal{H}_{M N}$. With $h=\left(h^{M}{ }_{N}\right)$ an $O(d, d)$ group element, we have $h \eta h^{t}=\eta$ and the Liealgebra valued infinitesimal parameter $\tau$ arises from $h \simeq$ $1+\tau$ and thus satisfies (3.17). The transformation $\delta_{\tau} \mathcal{S}$ above is the infinitesimal version of the finite transformation $\mathcal{S} \rightarrow \mathcal{S}^{\prime}=h \mathcal{S} h^{-1}$, again with $h \simeq 1+\tau$. Note that $\delta_{\tau} \mathcal{S}=-\mathcal{S} \delta_{\tau} \mathcal{S S}$ and thus the variation preserves $\mathcal{S}^{2}=\mathbf{1}$. The symmetry constraint on $\mathcal{S}$ (inherited from $\mathcal{H}=\mathcal{H}^{t}$ ) reads

$$
\mathcal{S} \eta-\eta \mathcal{S}^{t}=0,
$$

and is also preserved under the variation $\delta_{\tau} \mathcal{S}$.

We can compute the Noether charges $\mathcal{Q}$ by the usual trick of promoting the symmetry parameter to be local, $\tau \rightarrow \tau(t)$, and to compute the variation

$$
\delta_{\tau} I=\int \mathrm{d} t \operatorname{tr}(\dot{\tau} \mathcal{Q})=-\int \mathrm{d} t \operatorname{tr}(\tau \dot{\mathcal{Q}}) .
$$

An explicit computation of the variation of the action $I$ in (3.1) under $\delta_{\tau} \mathcal{S}$ follows quickly from the first line in (3.2) and gives

$$
\mathcal{Q}=e^{-\Phi} \sum_{k=1}^{\infty} \alpha^{\prime k-1} 4 k c_{k} \mathcal{S} \dot{\mathcal{S}}^{2 k-1}
$$

Since on-shell the variation (3.19) must vanish for arbitrary functions $\tau$, we conclude that on-shell $\dot{\mathcal{Q}}=0$. Thus $\mathcal{Q}$ is conserved, which may also be verified by a quick computation with (3.6), which gives $\dot{\mathcal{Q}}=-2 e^{-\Phi} \mathcal{S} E_{\mathcal{S}}$, and shows that the conservation of $\mathcal{Q}$ is equivalent to the equation of motion of $\mathcal{S}$. Moreover, $\mathcal{Q}$ takes values in the Lie algebra $\mathfrak{s} \mathfrak{o}(d, d)$ as one quickly sees that $\mathcal{Q} \eta+\eta \mathcal{Q}^{t}=0$, using (3.18) and its time derivative.

\section{FRIEDMANN EQUATIONS TO ALL ORDERS IN $\alpha^{\prime}$}

In this section we evaluate the equations of motion (3.10) for the spatial metric set equal to a scale factor times the Euclidean metric and with vanishing $b$-field. This yields the $\alpha^{\prime}$ corrected Friedmann equations. In the first subsection we write the $O(d, d)$ covariant fields in terms of conventional cosmological variables and show that the standard (string) Friedmann equations are recovered to zeroth order in $\alpha^{\prime}$. In the second subsection we give the $\alpha^{\prime}$ corrected equations. In the final subsection we show how to integrate these equations to arbitrary order in $\alpha^{\prime}$.

\section{A. Review of two-derivative equations}

We now specialize to the FLRW metric with curvature $k=0$ and vanishing $b$-field: we set $g_{i j}=a^{2}(t) \delta_{i j}$ and $b_{i j}=0$ in (2.4). Together with the definition of the $O(d, d)$ invariant dilaton in (2.5) we then have

$\mathcal{S}(t)=\left(\begin{array}{cc}0 & a^{2}(t) \\ a^{-2}(t) & 0\end{array}\right), \quad e^{-\Phi}=(a(t))^{d} e^{-2 \phi}$,

in terms of the scale factor $a(t)$ and the scalar dilaton $\phi(t)$. We next introduce the Hubble parameter

$$
H \equiv \frac{\dot{a}}{a} .
$$


The time derivatives of the dilaton $\Phi$ then become

$$
\dot{\Phi}=-d H+2 \dot{\phi}, \quad \ddot{\Phi}=-d \dot{H}+2 \ddot{\phi},
$$

where

$$
\dot{H}=-H^{2}+\frac{\ddot{a}}{a} .
$$

For the following applications it will be convenient to establish some relations for the field $\mathcal{S}(t)$ and its derivatives. First, $\mathcal{S}(t)$ is constrained and satisfies $\mathcal{S}^{2}=\mathbf{1}$, and is hence a "pseudocomplex" structure. Amusingly, its derivative is proportional to a complex structure:

$$
\dot{\mathcal{S}}=2 H \mathcal{J}, \quad \mathcal{J} \equiv\left(\begin{array}{cc}
0 & a^{2}(t) \\
-a^{-2}(t) & 0
\end{array}\right), \quad \mathcal{J}^{2}=\mathbf{- 1} .
$$

In turn, the derivative of $\mathcal{J}$ is proportional to $\mathcal{S}$ :

$$
\dot{\mathcal{J}}=2 H \mathcal{S} \text {. }
$$

Thus, the second time derivative of $\mathcal{S}$ can be expressed in terms of $\mathcal{S}$ and $\mathcal{J}$ :

$$
\ddot{\mathcal{S}}=2 \dot{H} \mathcal{J}+4 H^{2} \mathcal{S} .
$$

Let us now evaluate the two-derivative equations of motion following from the action $I_{0}$ for the above ansatz, with the aim to compare with the standard Friedmann equations that follow directly from the higher dimensional action for metric and dilaton. After setting $n(t)=1$, the equations of motion of the two-derivative theory are those in (3.10), keeping only $c_{1}=-\frac{1}{8}$ and setting all $c_{k>1}$ equal to zero:

$$
\begin{aligned}
\ddot{\mathcal{S}}+\dot{\mathcal{S}}^{2}-\dot{\Phi} \dot{\mathcal{S}} & =0, \quad(\mathcal{S}) \\
-2 \ddot{\Phi}+\dot{\Phi}^{2}-\frac{1}{8} \operatorname{tr} \dot{\mathcal{S}}^{2} & =0, \quad(\Phi) \\
\dot{\Phi}^{2}+\frac{1}{8} \operatorname{tr} \dot{\mathcal{S}}^{2} & =0 .
\end{aligned}
$$

Inserting the above expressions for $\mathcal{S}, \Phi$, and their derivatives in terms of the scale factor, these equations become

$$
\begin{aligned}
&(d-1)\left(\frac{\dot{a}}{a}\right)^{2}+\frac{\ddot{a}}{a}-2 \frac{\dot{a}}{a} \dot{\phi}=0, \quad(\mathcal{S}) \\
& d(d-1) H^{2}-4 d H \dot{\phi}+4 \dot{\phi}^{2}-4 \ddot{\phi}+2 d \frac{\ddot{a}}{a}=0, \quad(\Phi) \\
& d(d-1) H^{2}-4 d H \dot{\phi}+4 \dot{\phi}^{2}=0 . \quad(n)
\end{aligned}
$$

Replacing the second equation by the third minus the second, we find

$$
\begin{aligned}
(d-1)\left(\frac{\dot{a}}{a}\right)^{2}+\frac{\ddot{a}}{a}-2 \frac{\dot{a}}{a} \dot{\phi} & =0, \quad(\mathcal{S}) \\
-2 \ddot{\phi}+d \frac{\ddot{a}}{a} & =0, \quad(n)-(\Phi) \\
d(d-1) H^{2}-4 d H \dot{\phi}+4 \dot{\phi}^{2} & =0 . \quad(n)
\end{aligned}
$$

Using linear combinations of the above equations, it is easy to see that they are equivalent to the three standard (string) Friedmann equations, as, for instance, given in [37].

\section{B. $\alpha^{\prime}$ corrected equations}

Let us now turn to the computation of the $\alpha^{\prime}$ corrected Friedmann equations. We will start from the equations in (3.10) and specialize to the FLRW ansatz with $k=0$ shown in (4.1). Although these equations were derived only from the general single-trace action (3.1), we will now argue that for the FLRW ansatz, and leaving the coefficients $c_{k}$ generic, this is, in fact, the most general action.

Specifically, we will show that the inclusion of any multitrace invariants in the action merely renormalizes the coefficients $c_{k}$. To this end note that with $\dot{\mathcal{S}}=2 H \mathcal{J}$ and $\mathcal{J}^{2}=-\mathbf{1}$ we can immediately evaluate a generic term of order $\alpha^{\prime k-1}$ in the Lagrangian:

$$
\mathcal{L} \propto c_{k} \operatorname{tr}\left(\dot{\mathcal{S}}^{2 k}\right)=(-1)^{k} 2^{2 k+1} c_{k} d H^{2 k}(t) .
$$

Now, let us compare this with, say, a double trace term with the same order of $\alpha^{\prime}$ :

$\mathcal{L} \propto c_{k, l} \operatorname{tr}\left(\dot{\mathcal{S}}^{2(k-l)}\right) \operatorname{tr}\left(\dot{\mathcal{S}}^{2 l}\right)=c_{k, l}(-1)^{k} 2^{2 k+1} 2 d^{2} H^{2 k}(t)$,

where $c_{k, l}$ are new coefficients. Comparing with (4.11) we see that they coincide, except for the overall normalization. Thus, the only effect of the inclusion of (4.12) is the renormalization

$$
c_{k} \rightarrow c_{k}+2 d c_{k, l} .
$$

More generally, the inclusion of any multitrace invariant merely leads to a ( $d$-dependent) renormalization of the coefficients $c_{k}$. (We have also verified this at the level of the equations of motion.) Thus, it is sufficient to start with Eqs. (3.10) obtained from the single-trace action.

Let us begin with the $\mathcal{S}$ field equation. In order to evaluate it efficiently we first collect some relations for $\mathcal{S}$ and $\mathcal{J}$ that follow from $\dot{\mathcal{S}}=2 H \mathcal{J}$ and $\mathcal{J}^{2}=\mathbf{- 1}$ :

$$
\begin{aligned}
\dot{\mathcal{S}}^{2 k} & =(-1)^{k}(2 H)^{2 k} \mathcal{S}, \\
\dot{\mathcal{S}}^{2 k-1} & =\dot{\mathcal{S}}^{2(k-1)} \dot{\mathcal{S}}=(-1)^{k-1}(2 H)^{2 k-1} \mathcal{J} .
\end{aligned}
$$

We can then evaluate the derivative 


$$
\begin{aligned}
\frac{d}{d t} \dot{\mathcal{S}}^{2 k-1}= & (2 k-1)(-1)^{k-1}(2 H)^{2(k-1)} 2 \dot{H} \mathcal{J} \\
& +(-1)^{k-1}(2 H)^{2 k} \mathcal{S}
\end{aligned}
$$

and thus compute

$$
\begin{aligned}
& \frac{d}{d t} \dot{\mathcal{S}}^{2 k-1}-\dot{\Phi} \dot{\mathcal{S}}^{2 k-1}+\mathcal{S}^{2 k} \\
& =(-1)^{k-1}(2 H)^{2 k-2}((2 k-1) 2 \dot{H}-\dot{\Phi}(2 H)) \mathcal{J} \\
& =(-1)^{k-1} 2^{2 k-1}\left[(2 k-1) \dot{H} H^{2 k-2}-\dot{\Phi} H^{2 k-1}\right] \mathcal{J} \\
& =(-1)^{k-1} 2^{2 k-1}\left(\left[\frac{d}{d t}-\dot{\Phi}\right] H^{2 k-1}\right) \mathcal{J} .
\end{aligned}
$$

Note that here all terms proportional to $\mathcal{S}$ canceled. This means that one obtains only a single equation, not a matrix equation, as it should be, because we are looking for an equation for the single function $a(t)$. Writing $E_{\mathcal{S}}=\mathcal{E}_{\mathcal{S}} \mathcal{J}$ we find

$$
\begin{aligned}
\mathcal{E}_{\mathcal{S}} & =-\sum_{k=1}^{\infty}\left(-\alpha^{\prime}\right)^{k-1} k c_{k} 2^{2 k}\left[\frac{d}{d t}-\dot{\Phi}\right] H^{2 k-1} \\
& =-\left[\frac{d}{d t}-\dot{\Phi}\right] \sum_{k=1}^{\infty}\left(-\alpha^{\prime}\right)^{k-1} k c_{k} 2^{2 k} H^{2 k-1}
\end{aligned}
$$

The last expression suggests the definition of a function $f(H)$ of the Hubble parameter $H$. Including some overall constants for future convenience we write

$$
\begin{aligned}
f(H) & \equiv 4 d \sum_{k=1}^{\infty}\left(-\alpha^{\prime}\right)^{k-1} 2^{2 k} k c_{k} H^{2 k-1} \\
& =16 d c_{1} H-128 d \alpha^{\prime} c_{2} H^{3}+\cdots,
\end{aligned}
$$

for then Eqs. (4.17) can simply be written as

$$
\left(\frac{d}{d t}-\dot{\Phi}(t)\right) f(H)=0
$$

This drastic simplification of the equations can be understood as a consequence of the existence of the Noether charges (3.20): for the FLRW ansatz $\mathcal{Q}$ can be seen to be

$$
\mathcal{Q}=\frac{1}{2 d} e^{-\Phi(t)} f(H(t))\left(\begin{array}{cc}
-\mathbf{1} & 0 \\
0 & \mathbf{1}
\end{array}\right)
$$

The conservation law $\dot{\mathcal{Q}}=0$ gives $\frac{d}{d t}\left(e^{-\Phi} f(H)\right)=0$, which is equivalent to (4.19), as anticipated before.

We now determine the lapse and dilaton equations from (3.10). For the lapse equation one obtains
$\dot{\Phi}^{2}=-2 d \sum_{k=1}^{\infty}\left(-\alpha^{\prime}\right)^{k-1} 2^{2 k}(2 k-1) c_{k} H^{2 k}=-g(H)$,

where we introduced the function

$$
\begin{aligned}
g(H) & \equiv 2 d \sum_{k=1}^{\infty}\left(-\alpha^{\prime}\right)^{k-1} 2^{2 k}(2 k-1) c_{k} H^{2 k} \\
& =8 d c_{1} H^{2}-\alpha^{\prime} 3 \cdot 2^{5} d c_{2} H^{4}+\cdots
\end{aligned}
$$

The dilaton equation is most conveniently written in the combination (3.14) using the lapse equation. One finds

$$
0=\ddot{\Phi}+2 d \sum_{k=1}^{\infty}\left(-\alpha^{\prime}\right)^{k-1} 2^{2 k} k c_{k} H^{2 k} .
$$

On the right-hand side we recognize a multiple of $H f(H)$. Thus, the above equation can also be written as

$$
\ddot{\Phi}+\frac{1}{2} H f(H)=0 .
$$

Summarizing, the three $\alpha^{\prime}$-completed Friedmann equations take the form

$$
\begin{aligned}
\frac{d}{d t}\left(e^{-\Phi} f(H)\right) & =0, \\
\ddot{\Phi}+\frac{1}{2} H f(H) & =0, \\
\dot{\Phi}^{2}+g(H) & =0,
\end{aligned}
$$

in terms of the functions $f(H)$ and $g(H)$ defined in (4.18) and (4.22):

$$
\begin{aligned}
f(H) & =4 d \sum_{k=1}^{\infty}\left(-\alpha^{\prime}\right)^{k-1} 2^{2 k} k c_{k} H^{2 k-1}=-2 d H+\mathcal{O}\left(\alpha^{\prime}\right), \\
g(H) & =2 d \sum_{k=1}^{\infty}\left(-\alpha^{\prime}\right)^{k-1} 2^{2 k}(2 k-1) c_{k} H^{2 k} \\
& =-d H^{2}+\mathcal{O}\left(\alpha^{\prime}\right) .
\end{aligned}
$$

These definitions show that $f$ and $g$ are, in fact, closely related. One readily verifies that

$$
g^{\prime}(H)=H f^{\prime}(H),
$$

where ' denotes differentiation with respect to $H$.

Let us discuss briefly some general properties of its solutions. First, as expected, they are invariant under the duality transformation $a \rightarrow \frac{1}{a}$. Indeed, under this transformation

$H \rightarrow-H, \quad \Phi \rightarrow \Phi, \quad f(H) \rightarrow-f(H)$,

$g(H) \rightarrow g(H)$, 
which leaves Eqs. (4.25) invariant. Thus, for any given solution $a(t)$ there is a "dual" solution $\tilde{a}(t) \equiv 1 / a(t)$.

Equations (4.25) are also invariant under time reversal $t \rightarrow-t$. For a given solution $a(t), \Phi(t)$, there is the timereversed solution $\tilde{a}(t) \equiv a(-t), \tilde{\Phi}(t) \equiv \Phi(-t)$, with $\tilde{H}(t)=$ $-H(-t)$.

\section{Perturbative solutions}

We now discuss how to solve the $\alpha^{\prime}$-corrected Friedmann equations (4.25) perturbatively in $\alpha^{\prime}$. To this end it is convenient to introduce the variable $\Omega$ as an exponential of the dilaton $\Phi$ :

$$
\Omega \equiv e^{-\Phi} .
$$

Using the derivatives $\dot{\Omega}=-\dot{\Phi} \Omega$ and $\ddot{\Omega}=\left(-\ddot{\Phi}+\dot{\Phi}^{2}\right) \Omega$, and taking the difference of the second and third equations in (4.25), the set of equations becomes

$$
\begin{aligned}
X & \equiv \frac{d}{d t}(\Omega f(H))=0, \\
Y & \equiv \ddot{\Omega}-h(H) \Omega=0, \\
Z & \equiv \dot{\Omega}^{2}+g(H) \Omega^{2}=0,
\end{aligned}
$$

where, for later use, they have been labeled $X, Y, Z$, and we defined the function $h(H)$ as

$$
\begin{aligned}
h(H) & \equiv \frac{1}{2} H f(H)-g(H) \\
& =-2 d \sum_{k=2}^{\infty}\left(-\alpha^{\prime}\right)^{k-1} 2^{2 k}(k-1) c_{k} H^{2 k} \\
& =\alpha^{\prime} 2^{5} d c_{2} H^{4}+\cdots .
\end{aligned}
$$

Note that $h$, in contrast to $f$ and $g$, vanishes to zeroth order in $\alpha^{\prime}$, which will be crucial for our subsequent perturbative construction. It will also be crucial to recall that there is one Bianchi identity among the three equations; cf. (3.13). For the functions defined in (4.30) the Bianchi identity takes the form

$$
\frac{d Z}{d t}=H \Omega X+2 \dot{\Omega} Y .
$$

This is easily verified directly by differentiation recalling that $g^{\prime}(H)=H f^{\prime}(H)$.

We will now show how to solve these equations, perturbatively in $\alpha^{\prime}$, by expanding

$\Omega(t)=\Omega_{0}(t)+\alpha^{\prime} \Omega_{1}(t)+\left(\alpha^{\prime}\right)^{2} \Omega_{2}(t)+\cdots$,

$H(t)=H_{0}(t)+\alpha^{\prime} H_{1}(t)+\left(\alpha^{\prime}\right)^{2} H_{2}(t)+\cdots$.

The subscripts in these quantities denote the power of $\alpha^{\prime}$ that accompanies them in the expansion. The first equation, $X=0$, is the Noether conservation, solved once and for all by

$$
f(H(t))=q \Omega^{-1}(t),
$$

with $q$ a number, proportional to the Noether charge $\mathcal{Q}$ [see (4.20)]. The question arises whether integration constants such as $q$ have to be treated also as expansions in $\alpha^{\prime}$ by writing $q=q_{0}+\alpha^{\prime} q_{1}+\cdots$. This depends on how we set out to solve the differential equations. If we pose initial conditions, say by specifying $\Omega(0)$ and $\dot{\Omega}(0)$, then we have to determine all integration constants in terms of these initial data, in which case the value of $q$, for example, does get $\alpha^{\prime}$ corrected. Here, however, we will follow a different prescription that simplifies the analysis. Instead of formulating an explicit initial value problem we introduce integration constants whenever necessary, but allow ourselves the freedom to set integration constants to zero whose only effect is to "renormalize" constants that are already present. As long as these previously introduced integration constants are completely general, this procedure does not entail a loss of generality. Then there is no need for $\alpha^{\prime}$ expansions of parameters such as $q$.

We now show how to iteratively solve the above equations to any desired order in $\alpha^{\prime}$. Since $h(H)$ starts at order $\alpha^{\prime}$, to lowest order $Y=0$ implies

$$
\ddot{\Omega}_{0}(t)=0 \quad \Rightarrow \quad \Omega_{0}(t)=\gamma\left(t-t_{0}\right),
$$

which we solved in terms of two integration constants $\gamma$ and $t_{0}$. Then, from the expansion of $f(H)$ and (4.34),

$$
\begin{aligned}
f(H) & \equiv 16 d c_{1} H_{0}=q \frac{1}{\gamma\left(t-t_{0}\right)} \Rightarrow \\
H_{0}(t) & =-\frac{q}{2 d \gamma} \frac{1}{t-t_{0}},
\end{aligned}
$$

where we used the universal $c_{1}=-\frac{1}{8}$. The last equation of (4.30), $Z=0$, can now be used to to zeroth order in $\alpha^{\prime}$ to establish a relation between $q$ and the other parameters:

$0=\dot{\Omega}_{0}^{2}+g(H) \Omega_{0}^{2}=\gamma^{2}-d H_{0}^{2}(t) \gamma^{2}\left(t-t_{0}\right)^{2}=\gamma^{2}-\frac{q^{2}}{4 d}$,

and thus

$$
q= \pm 2 \sqrt{d}|\gamma| .
$$

The solutions are then summarized by

$H_{0}(t)=\mp \frac{\operatorname{sgn}(\gamma)}{\sqrt{d}} \frac{1}{t-t_{0}}, \quad \Omega_{0}(t)=\gamma\left(t-t_{0}\right)$.

This is the complete solution to zeroth order in $\alpha^{\prime}$ that is well defined for $t>t_{0}$. Before turning to next order, let us express this solution in terms of standard variables. From the definition $H(t)=\frac{d}{d t} \ln (a(t))$ we obtain by integration 


$$
\ln (a(t))=\mp \frac{\operatorname{sgn}(\gamma)}{\sqrt{d}} \ln \left(t-t_{0}\right)+\text { const }
$$

and thus

$$
a(t)=A\left(t-t_{0}\right)^{\mp \operatorname{sgn}(\gamma) \frac{1}{\sqrt{d}}},
$$

where $A$ is a new integration constant. For the scalar dilaton this yields with (4.1)

$$
\begin{aligned}
e^{-2 \phi} & =(a(t))^{-d} e^{-\Phi}=(a(t))^{-d} \Omega_{0} \\
& \propto\left(t-t_{0}\right)^{1 \pm \operatorname{sgn}(\gamma) \sqrt{d}} .
\end{aligned}
$$

This solution is well known in the literature [see Eqs. (4.1)(4.4) in [29]], which, for $d=25$ and the special case that all radii are equal, reduces to (4.41) and (4.42) for $\gamma<0$. See also [32] for first-order $\alpha^{\prime}$ corrections. (The relation to our results is more difficult to establish due to the ambiguity in field variables and their truncation to four derivatives.)

Let us now turn to the solutions of first order in $\alpha^{\prime}$. To this order, the equation $Y=0$ implies

$$
\begin{aligned}
\alpha^{\prime} \ddot{\Omega}_{1} & =h(H) \Omega_{0}=d\left(\alpha^{\prime} 2^{5} c_{2} H_{0}^{4}(t)\right) \Omega_{0} \\
& =\alpha^{\prime} d 2^{5} c_{2} \frac{1}{d^{2}} \frac{1}{\left(t-t_{0}\right)^{4}} \gamma\left(t-t_{0}\right),
\end{aligned}
$$

and so we need to solve

$$
\ddot{\Omega}_{1}=c_{2} 2^{5} \frac{\gamma}{d} \frac{1}{\left(t-t_{0}\right)^{3}} .
$$

This can be integrated immediately:

$$
\Omega_{1}(t)=c_{2} 2^{4} \frac{\gamma}{d} \frac{1}{t-t_{0}}+b_{1} t+b_{2},
$$

where $b_{1}$ and $b_{2}$ are integration constants. Looking back at the zeroth-order solution (4.39) we infer that these effectively "renormalize" the previous integration constants $\gamma$ and $t_{0}$. Thus, without loss of generality we can set $b_{1}=$ $b_{2}=0$ :

$$
\Omega_{1}(t)=\frac{2^{4} c_{2} \gamma}{d} \frac{1}{t-t_{0}} .
$$

Next, we have to determine $H_{1}(t)$. To this end we use (4.34) and expand to first order in $\alpha^{\prime}$ :

$$
\begin{aligned}
f(H) & =q \Omega^{-1}=q \Omega_{0}^{-1}\left(1+\alpha^{\prime} \Omega_{0}^{-1} \Omega_{1}\right)^{-1} \\
& =q\left(\Omega_{0}^{-1}-\alpha^{\prime} \frac{\Omega_{1}}{\Omega_{0}^{2}}\right)
\end{aligned}
$$

which then has to be matched to the definition of $f$ expanded to the same order,

$$
\begin{aligned}
f(H) & =16 d c_{1} H-128 d \alpha^{\prime} c_{2} H^{3} \\
& =16 d c_{1} H_{0}+16 d \alpha^{\prime}\left(c_{1} H_{1}-8 c_{2} H_{0}^{3}\right) .
\end{aligned}
$$

Thus, to first order in $\alpha^{\prime}$ we have to solve

$$
-\frac{q}{16 d} \frac{\Omega_{1}}{\Omega_{0}^{2}}=c_{1} H_{1}-8 c_{2} H_{0}^{3} .
$$

Using (4.39) and (4.46) this can be solved for $H_{1}$,

$$
H_{1}(t)= \pm \frac{80 \operatorname{sgn}(\gamma) c_{2}}{d^{3 / 2}} \frac{1}{\left(t-t_{0}\right)^{3}}
$$

Given the way we have set up the computation, the $Z=0$ equation now needs to be satisfied identically, for we have no free parameters left to fix by this equation. An explicit computation to first order in $\alpha^{\prime}$ shows that $Z=0$ is indeed satisfied.

More generally, it is clear that the iterative procedure of solving the equations can be continued to arbitrary orders as follows. Consider first the equation $Y=0$, setting equal the terms that are of order $\alpha^{\prime k}$ :

$$
\alpha^{\prime k} \ddot{\Omega}_{k}=(h(H) \Omega)_{k} .
$$

Suppose we have solved for $\Omega_{0}, \ldots, \Omega_{k-1}$, as well as for $H_{0}, \ldots, H_{k-1}$. Since $h(H)$ starts at order $\alpha^{\prime}$, the right-hand side only involves $\Omega_{i}$ 's and $H_{i}$ 's that are already determined. Thus $\ddot{\Omega}_{k}$ is determined, and we can directly integrate twice to determine $\Omega_{k}$. This gives two integration constants as in (4.45), but as before these just renormalize $\gamma, t_{0}$ and so we set them to zero. With $\Omega_{0}, \ldots, \Omega_{k}$ now determined we next use the equation $X=0$ in the form (4.34) in order to determine $H_{k}$, which completes the next iteration step. Upon following the structural dependence on the time-dependent factor $\left(t-t_{0}\right)$ in this computation one may, in fact, verify that the perturbative solutions take the form

$$
\begin{aligned}
& H(t)=h_{0} \frac{1}{t-t_{0}}+\alpha^{\prime} h_{1} \frac{1}{\left(t-t_{0}\right)^{3}}+\alpha^{\prime 2} h_{2} \frac{1}{\left(t-t_{0}\right)^{5}}+\cdots, \\
& \Omega(t)=\omega_{0}\left(t-t_{0}\right)+\alpha^{\prime} \omega_{1} \frac{1}{t-t_{0}}+\alpha^{\prime 2} \omega_{2} \frac{1}{\left(t-t_{0}\right)^{3}}+\cdots,
\end{aligned}
$$

where $h_{n}$ and $\omega_{n}$ are time-independent coefficients, which can in principle be expressed in terms of the $c_{n}$, as done above for the first two. Finally, it remains to prove that the $Z=0$ equation is automatically satisfied. To this end we first note that the above expansions of $H$ and $\Omega$ in (4.52) determine the time dependence of $Z=\dot{\Omega}^{2}+g(H) \Omega^{2}$ to be of the form 
$Z(t)=z_{0}+\alpha^{\prime} z_{1} \frac{1}{\left(t-t_{0}\right)^{2}}+\alpha^{\prime 2} z_{2} \frac{1}{\left(t-t_{0}\right)^{4}}+\cdots$,

where the $z_{i}$ are time-independent parameters. Now, since we have satisfied $X=Y=0$ in finding (4.52), the Bianchi identity (4.32) implies that $Z$ does not depend on time, so actually $z_{1}=z_{2}=\cdots=0$. Therefore, $Z(t)=z_{0}$, but since we fixed parameters in (4.37) so that $z_{0}=0$, we have established $Z=0$ to all orders in $\alpha^{\prime}$.

We close this section with a few remarks. The above solutions (4.52) seem to suggest that the perturbative expansion breaks down in the high curvature region, since the terms become more and more singular as $t \rightarrow t_{0}$. However, it is conceivable that the entire series converges to a regular function (as, for instance, $e^{-\frac{1}{t-t_{0}}}$ is regular at $t \rightarrow t_{0}$ although every term in its power series diverges). Moreover, for late times $t$ the solutions seem to imply that $H$ is driven to zero and thus to Minkowski space, but this, of course, may change once matter contributions are included. In any case, it is important to investigate the possible nonperturbative solutions, to which we turn in the next section. ${ }^{2}$

\section{NONPERTURBATIVE SOLUTIONS}

In this section we discuss a few aspects of possible solutions that are nonperturbative in $\alpha^{\prime}$. In the first subsection we solve the initial-value formulation for the general equations derived above, for generic functions $f(H)$ and $g(H)$. In the second subsection we give conditions on the functions $f(H)$ and $g(H)$ so that the resulting theory permits de Sitter vacua.

\section{A. Initial-value formulation}

Let us now show how to obtain the general solution given initial conditions at time $t=0$ of the form

$$
\Omega(0)=\omega, \quad \dot{\Omega}(0)=\frac{1}{\sqrt{\alpha^{\prime}}} \gamma .
$$

Note that since $\Omega=e^{-\Phi}$ is unit free, $\omega$ and $\gamma$ are unit-free constants. Our strategy will be to solve the first and last equations in (4.30),

$$
\begin{aligned}
& X \equiv \frac{d}{d t}(\Omega f(H))=0, \\
& Z \equiv \dot{\Omega}^{2}+g(H) \Omega^{2}=0 .
\end{aligned}
$$

The equation $Y=0$ will then hold due to the Bianchi identity (4.32),

$$
\frac{d Z}{d t}=H \Omega X+2 \dot{\Omega} Y
$$

\footnotetext{
${ }^{2}$ We thank Robert Brandenberger and Jean-Luc Lehners for discussions on these points.
}

assuming $\dot{\Omega} \neq 0$ as we will do from now on. The case $\dot{\Omega}=0$ will be treated separately and is a bit singular.

Let us first see that the initial conditions fix the value $H(0)$ of the Hubble parameter at $t=0$. For this consider the second equation in (5.2) which at $t=0$ gives

$$
\alpha^{\prime} g(H(0))=-\frac{\gamma^{2}}{\omega^{2}}
$$

Note that we could pass to a new function $\tilde{g}$ defined so that

$\alpha^{\prime} g(H)=\tilde{g}\left(\sqrt{\alpha^{\prime}} H\right)=-d\left(\sqrt{\alpha^{\prime}} H\right)^{2}+\mathcal{O}\left(\left(\sqrt{\alpha^{\prime}} H\right)^{4}\right)$,

which is a unit-free function of the unit-free argument $\sqrt{\alpha^{\prime}} H$. Equation (5.4) then becomes

$$
\tilde{g}\left(\sqrt{\alpha^{\prime}} H(0)\right)=-\frac{\gamma^{2}}{\omega^{2}} .
$$

The possible values of $H(0)$ are the roots of this equation. In this way $\Omega(0)$ and $\dot{\Omega}(0)$ have determined $H(0)$ up to a discrete ambiguity. Equation (5.5) makes it clear that we can always solve perturbatively for $H(0)$ but, of course, it could be that nonperturbatively there are no solutions.

Now that we have the initial value of $H$ we use the $X=0$ equation to solve for $\dot{\Omega} / \Omega$ as a function of $H$ :

$$
\frac{\dot{\Omega}}{\Omega}=-\frac{f^{\prime}(H)}{f(H)} \dot{H} .
$$

The $Z=0$ equation can be rewritten as

$$
\frac{\dot{\Omega}}{\Omega}= \pm \sqrt{-g(H)}
$$

The last two equations imply

$$
\frac{f^{\prime}(H)}{f(H)} \dot{H}=\mp \sqrt{-g(H)},
$$

which can be rewritten as

$$
\frac{f^{\prime}(H) d H}{f(H) \sqrt{-g(H)}}=\mp d t
$$

Integrating from $t=0$ to $t$ then gives

$$
\int_{H(0)}^{H(t)} \frac{f^{\prime}(H) d H}{f(H) \sqrt{-g(H)}}=\mp t .
$$

This is a complete solution. If the integral is done by finding a $W(H)$ such that 
$d W(H)=\frac{f^{\prime}(H) d H}{f(H) \sqrt{-g(H)}}=-\frac{2}{H f(H)} d \sqrt{-g(H)}$,

where the second form follows by $g^{\prime}=H f^{\prime}$ [cf. (4.27)], the solution is of the form

$$
W(H(t))-W(H(0))=\mp t .
$$

This implicitly determines $H$ as a function of $t$. The value of $\Omega(t)$ now follows from the $X=0$ equation in that

$$
\Omega(t)=\frac{q}{f(H(t))}=\omega \frac{f(H(0))}{f(H(t))},
$$

where in the second step the constant of the motion $q$ was evaluated using the initial condition $\Omega(0)=\omega$ and the value of $H(0)$.

Consider now the somewhat singular case of vanishing $\dot{\Omega}$. If $\dot{\Omega} \equiv 0$ for all $t$, the $Z=0$ equation implies $g(H)=0$, which fixes $H(t)$ to be a constant equal to a zero of $g$. We will consider this case in the next subsection. If $t=0$ is the only time for which $\dot{\Omega}=0$, then this fixes the initial condition for $H$.

\section{B. Nonperturbative de Sitter solutions}

We now turn to a discussion of possible de Sitter (dS) solutions. To this end consider the dS metric

$$
d s^{2}=-d t^{2}+e^{2 H_{0} t} d \mathbf{x}^{2}
$$

leading to the scale factor $a(t)=e^{H_{0} t}$, with $H=H_{0} \neq 0$ const. Consider the Friedmann equations (4.25) copied here for convenience:

$$
\begin{aligned}
\frac{d}{d t}\left(e^{-\Phi} f(H)\right) & =0, \\
\ddot{\Phi}+\frac{1}{2} H f(H) & =0, \\
\dot{\Phi}^{2}+g(H) & =0 .
\end{aligned}
$$

With $H$ constant, the first equation then implies that $\Phi$ is also constant:

$$
\dot{\Phi}=0 \rightarrow \Phi=\text { const }
$$

Since $H_{0} \neq 0$, the second equation implies the vanishing of $f\left(H_{0}\right)$ and the third the vanishing of $g\left(H_{0}\right)$ :

$$
f\left(H_{0}\right)=0, \quad g\left(H_{0}\right)=0 .
$$

Note that while the two functions $f(H)$ and $g(H)$ are different, $f(H)$ determines $g(H)$ via the relations $g^{\prime}(H)=$ $H f^{\prime}(H)$ and $g(0)=0$. In the two-derivative approximation
$f\left(H_{0}\right)=-2 d H_{0}$ [see (4.26)] and therefore the only solution is $H_{0}=0$, which is not of interest.

There are, however, nonperturbative dS solutions consistent with duality. That is, as we demonstrate below, there exist functions $f(H)$ such that $\mathrm{dS}$ solutions exist. To find a solution to (5.18) we integrate the relation $g^{\prime}(H)=H f^{\prime}(H)$ to find

$$
g(H)=H f(H)-\int_{0}^{H} f\left(H^{\prime}\right) \mathrm{d} H^{\prime},
$$

where we used $g(0)=0$, as required by the definition of $g$. To get a vanishing $g\left(H_{0}\right)$ when $f\left(H_{0}\right)=0$ we need that the integral of $f(H)$ from zero to $H_{0}$ vanishes. This, for example, happens at the nonvanishing zeros of the sine function. More explicitly, we take

$$
\begin{aligned}
f(H) & \equiv-\frac{2 d}{\sqrt{\alpha^{\prime}}} \sin \left(\sqrt{\alpha^{\prime}} H\right) \\
& =-2 d \sum_{k=1}^{\infty}\left(-\alpha^{\prime}\right)^{k-1} \frac{1}{(2 k-1) !} H^{2 k-1} .
\end{aligned}
$$

This $f(H)=-2 d H+\mathcal{O}\left(\alpha^{\prime}\right)$ is consistent with the known two-derivative theory. From the definition (4.18) it is clear that there are coefficients $c_{k}$ so that this holds (in fact, $\left.c_{k}=-\frac{1}{(2 k !) 2^{2 k}}\right)$. With (5.19) we then get

$g(H)=-\frac{2 d}{\alpha^{\prime}}\left(\sqrt{\alpha^{\prime}} H \sin \left(\sqrt{\alpha^{\prime}} H\right)+\cos \left(\sqrt{\alpha^{\prime}} H\right)-1\right)$.

We now see that $f\left(H_{0}\right)=g\left(H_{0}\right)=0$ is satisfied for

$$
\sqrt{\alpha^{\prime}} H_{0}=2 \pi n, \quad n \in \mathbb{Z}, \quad n \neq 0 .
$$

This is a discrete infinity of dS solutions.

Although (5.20) is surely not the function arising in any string theory, the actual functions could have the properties required in order to lead to dS vacua in the string frame. The conditions for this to happen can be stated more concisely as follows. Instead of $f(H)$ we consider its integral

$F(H) \equiv \int_{0}^{H} f\left(H^{\prime}\right) d H^{\prime}=4 d \sum_{k=1}^{\infty}\left(-\alpha^{\prime}\right)^{k-1} 2^{2 k-1} c_{k} H^{2 k}$,

where the evaluation used the expansion (4.26) of $f(H)$. Then, from (5.19), we have that $g(H)$ is given by

$$
g(H)=H F^{\prime}(H)-F(H) .
$$

We now infer that if there is an $H_{0} \neq 0$ so that $F$ and its first derivative vanish at this point, 


$$
F\left(H_{0}\right)=F^{\prime}\left(H_{0}\right)=0,
$$

then $H_{0}$ is a zero of both $f$ and $g$, and hence the theory permits dS solutions. The conditions above require $F$ to attain value zero at maxima or minima. Such functions are easily constructed, as we will show below. Since the leading term in $f(H)$ is fixed by the two-derivative theory, we also have $F(H)=-d H^{2}+\mathcal{O}\left(\alpha^{\prime}\right)$.

Let us emphasize that the $\mathrm{dS}$ solutions discussed above are nonperturbative in $\alpha^{\prime}$ : they are not constructed from a solution of the two-derivative equations which is then $\alpha^{\prime}$ corrected. The complete series of all $\alpha^{\prime}$ corrections needs to be used in order to obtain a solution.

We demonstrated that the two-derivative theory does not have $\mathrm{dS}$ solutions. The inclusion of the leading $\alpha^{\prime}$ corrections does not change this. But with suitable order $\alpha^{\prime 2}$ corrections, dS solutions appear. This corresponds to $f(H) \sim H+\alpha^{\prime} H^{3}+\alpha^{\prime 2} H^{5}$, and therefore $F(H) \sim H^{2}+$ $\alpha^{\prime} H^{4}+\alpha^{\prime 2} H^{6}$. It is simple to see that this happens for

$$
F(H)=-d H^{2}\left(1-\gamma^{2} \alpha^{\prime} H^{2}\right)^{2},
$$

where $\gamma$ is an arbitrary constant. The solution here is $\sqrt{\alpha^{\prime}} H_{0}= \pm 1 / \gamma$. Such solutions, however curious, cannot be trusted in string theory, which carries an infinite number of $\alpha^{\prime}$ corrections. A reliable solution should either solve the two-derivative equations (and then be corrected perturbatively) or be nonperturbative in $\alpha^{\prime}$.

A general class of functions $F(H)$ admitting dS solutions is easily obtained. For a polynomial $w(x)$ the condition $w\left(x_{0}\right)=0$ means that $w(x)=\left(x-x_{0}\right) u(x)$, where $u(x)$ is another polynomial. The additional condition $w^{\prime}\left(x_{0}\right)=0$ implies that $u\left(x_{0}\right)=0$, and thus $w(x)=\left(x-x_{0}\right)^{2} v(x)$ for some polynomial $v(x)$. While $F(H)$ is an infinite series in $H^{2}$, not a polynomial, the above remarks show how to produce consistent solutions. A general class of $F(H)$ with possible dS Hubble parameters $\pm H_{0}^{(1)}, \ldots, \pm H_{0}^{(k)}$ takes the form

$F(H)=-d H^{2}\left(1+\sum_{p=1}^{\infty} d_{p} \alpha^{\prime p} H^{2 p}\right) \prod_{i=1}^{k}\left(1-\left(\frac{H}{H_{0}^{(i)}}\right)^{2}\right)^{2}$.

The first factor in parentheses is an arbitrary series in $H^{2}$ with a leading term equal to one.

We close with a few general remarks on the possible dS solutions. First, they are most likely insufficient as phenomenologically viable models, because the natural values of the cosmological constant $\Lambda \sim 1 / \alpha^{\prime}$ obtained by this mechanism are many orders of magnitude too large. This is the cosmological constant problem. Second, they are dS solutions in the string frame, while the match with the observable dark energy is conventionally done in the
Einstein frame. We will now discuss some aspects of the Einstein-frame equations. ${ }^{3}$

Denoting the Einstein frame metric by $G_{\mu \nu}$, the required Weyl rescaling reads

$$
G_{\mu \nu}=e^{-\frac{4 \phi}{D-2}} g_{\mu \nu}, \quad D=d+1,
$$

which implies for the Einstein frame scale factor $a_{E}(t)=$ $e^{-\frac{2}{d-1} \phi(t)} a(t)$. Thus, the Einstein frame Hubble parameter reads

$H_{E} \equiv \frac{\dot{a}_{E}(t)}{a_{E}(t)}=-\frac{2}{d-1} \dot{\phi}+H=-\frac{1}{d-1}(\dot{\Phi}+H)$,

where we used the relation (4.3) between dilaton derivatives:

$$
2 \dot{\phi}=\dot{\Phi}+d H .
$$

We next have to recall that the Weyl rescaling (5.28) implies

$$
G_{00}=e^{-\frac{4 \phi}{D-2}} g_{00}=-e^{-\frac{4 \phi}{D-2}},
$$

since we have set $g_{00}=-n^{2}=-1$. To identify a de Sitter solution in the standard form (5.15) we need $G_{00}=-1$, and hence we need to reparametrize time. Let $t^{\prime}(t)$ be a time such that $G_{00}^{\prime}\left(t^{\prime}\right)=-1$ and thus

$G_{00}(t) d t^{2}=G_{00}^{\prime}\left(t^{\prime}\right) d t^{\prime 2}=-d t^{\prime 2} \rightarrow e^{-\frac{2 \phi}{d-1}} d t=d t^{\prime}$.

This redefinition has no effect on the $g_{i j}$ components of the metric other than reparametrizing the time dependence, so we have

$$
a_{E}^{\prime}\left(t^{\prime}\right)=a_{E}\left(t\left(t^{\prime}\right)\right) .
$$

Therefore the Hubble parameter of the solution in the Einstein frame with coordinates $\left(t^{\prime}, x^{i}\right)$ is

$$
\begin{aligned}
H_{E}^{\prime}\left(t^{\prime}\right) & =\frac{1}{a_{E}^{\prime}\left(t^{\prime}\right)} \frac{d a_{E}^{\prime}\left(t^{\prime}\right)}{d t^{\prime}}=\frac{d t}{d t^{\prime}} \cdot \frac{1}{a_{E}(t)} \frac{d a_{E}(t)}{d t}=e^{\frac{2 \phi}{d-1}} H_{E}\left(t\left(t^{\prime}\right)\right) \\
& =-e^{\frac{2 \phi}{d-1}} \frac{1}{d-1}(\dot{\Phi}+H),
\end{aligned}
$$

where we used $H_{E}(t)$ computed in (5.29). On the righthand side dot is a $t$ derivative, and all terms are evaluated at $t\left(t^{\prime}\right)$. Since $e^{2 \phi}=(a(t))^{d} e^{\Phi}$, we then have

$$
H_{E}^{\prime}\left(t^{\prime}\right)=-(a(t))^{\frac{d}{d-1}} e^{\frac{\Phi}{d-1}} \frac{1}{d-1}(\dot{\Phi}+H) .
$$

Our solutions with $\dot{\Phi}=0$ and $H=H_{0}$ would lead to a time-dependent $H_{E}^{\prime}$ through the scale factor $a(t) \sim e^{H_{0} t}$.

\footnotetext{
${ }^{3}$ We thank M. Gasperini and G. Veneziano for explanations on how to relate the two frames.
} 
Thus, these solutions do not lead to dS vacua in the Einstein frame.

The general condition for $\mathrm{dS}$ vacua in the Einstein frame is $\frac{d}{d t^{\prime}} H_{E}^{\prime}\left(t^{\prime}\right)=0$ and thus, by the chain rule, that the right-hand side of (5.34) or (5.35) is independent of $t$. We have not investigated the general problem whether there are solutions with this property, but as an illustration for the usefulness of this framework we present a simple no-go result: there are no dS solutions in the Einstein frame with constant dilaton $\phi$. To this end we observe that $\dot{\phi}=0$ implies with (5.30) that $\dot{\Phi}=-d H$, which with (5.34) yields

$$
H_{E}^{\prime}\left(t^{\prime}(t)\right)=e^{\frac{2 \phi}{d-1}} H(t) .
$$

Since we assume $\phi$ to be constant, $H_{E}^{\prime}$ is constant if and only if $H$ is constant. With $H$ constant, however, we are back to our previous analysis of $\mathrm{dS}$ vacua in the string frame, where we concluded that the Friedmann equations (5.16) imply $\dot{\Phi}=0$ and hence, recalling $\dot{\Phi}=-d H$, that $H=0$, leading to $H_{E}^{\prime}=0$ and flat space in the Einstein frame. This proves that there are no dS vacua in the Einstein frame with constant $\phi$.

\section{C. de Sitter solutions in noncritical string theory}

We now briefly comment on the general case of noncritical string theory in arbitrary dimension $D$ in which case the last term in (2.1) needs to be included. Note that this term is not a cosmological constant term since it is written in string frame. Rather, passing to the Einstein frame, this term gives rise to a scalar potential for $\phi$. The theory including at most two derivatives still does not allow for de Sitter vacua, as we confirm momentarily.

Let us now analyze the dynamics of FLRW backgrounds in noncritical string theory. As argued around (4.11) it is sufficient to include single-trace invariants, and so we consider the action that simply extends (3.1) by the additional term (in the gauge $n=1$ ):

$I \equiv \int \mathrm{d} t e^{-\Phi}\left(-\dot{\Phi}^{2}+\sum_{k=1}^{\infty} \alpha^{\prime k-1} c_{k} \operatorname{tr}\left(\dot{\mathcal{S}}^{2 k}\right)-\frac{2(D-26)}{3 \alpha^{\prime}}\right)$.

The equations of motion (3.10) are unaffected for $\mathcal{S}$, but are modified for $\Phi$ and $n$ :

$$
\begin{aligned}
& E_{\Phi} \equiv 2 \ddot{\Phi}-\dot{\Phi}^{2}+\frac{2(D-26)}{3 \alpha^{\prime}}-\sum_{k=1}^{\infty} \alpha^{\prime k-1} c_{k} \operatorname{tr}\left(\dot{\mathcal{S}}^{2 k}\right), \\
& E_{\mathcal{S}} \equiv-2 \sum_{k=1}^{\infty} \alpha^{\prime k-1} k c_{k}\left(\frac{d}{d t} \dot{\mathcal{S}}^{2 k-1}-\dot{\Phi}^{2 k-1}+\mathcal{S}^{2 k}\right), \\
& E_{n} \equiv \dot{\Phi}^{2}-\frac{2(D-26)}{3 \alpha^{\prime}}-\sum_{k=1}^{\infty} \alpha^{\prime k-1}(2 k-1) c_{k} \operatorname{tr}\left(\dot{\mathcal{S}}^{2 k}\right) .
\end{aligned}
$$

Note that while $E_{\Phi}$ and $E_{n}$ are modified, their sum is not and is still given by (3.15). It is then straightforward to specialize these equations to the FLRW background:

$$
\begin{aligned}
\frac{d}{d t}\left(e^{-\Phi} f(H)\right) & =0, \\
\ddot{\Phi}+\frac{1}{2} H f(H) & =0, \\
\dot{\Phi}^{2}+g(H)-\frac{2(D-26)}{3 \alpha^{\prime}} & =0,
\end{aligned}
$$

which generalizes (5.16).

We can now state the conditions for de Sitter vacua for which $H=H_{0}=$ const. As before, we see that in the twoderivative theory there are no de Sitter vacua: the first equation of (5.39) implies that $\Phi$ is constant, $\dot{\Phi}=0$, which with $f(H)=-2 d H$ and the second equation implies that $H_{0}=0$, leading to flat space. Including all $\alpha^{\prime}$ corrections, the condition that (5.39) permits de Sitter solutions can be stated in terms of the function $F(H)$ defined in (5.23):

$$
F\left(H_{0}\right)=-\frac{2(D-26)}{3 \alpha^{\prime}}, \quad F^{\prime}\left(H_{0}\right)=0 .
$$

Indeed, one then finds

$$
\begin{aligned}
& f\left(H_{0}\right)=F^{\prime}\left(H_{0}\right)=0, \\
& g\left(H_{0}\right)=H_{0} F^{\prime}\left(H_{0}\right)-F\left(H_{0}\right)=\frac{2(D-26)}{3 \alpha^{\prime}},
\end{aligned}
$$

so that (5.39) is satisfied for constant $\Phi$. Thus, duality invariant theories with de Sitter vacua exist provided there is a function $F(H)=-d H^{2}+\mathcal{O}\left(H^{4}\right)$ with an extremum at a nonzero $H_{0}$ where it takes value $-\frac{2(D-26)}{3 \alpha^{\prime}}$. Clearly, these conditions can be met. As a toy example we display a quartic polynomial with these properties:

$$
F(H)=-d H^{2}\left(1-\frac{1}{2}\left(\frac{H}{H_{0}}\right)^{2}\right),
$$

for which

$F\left(H_{0}\right)=-\frac{d H_{0}^{2}}{2}, \quad F^{\prime}(H)=-2 d H\left(1-\left(\frac{H}{H_{0}}\right)^{2}\right)$,

so that (5.40) holds for $\sqrt{\alpha^{\prime}} H_{0}=2 \sqrt{\frac{d-25}{3 d}}$.

\section{CONCLUSIONS AND OUTLOOK}

In this paper we have discussed, to all orders in $\alpha^{\prime}$, the most general duality-invariant spacetime actions for a metric, $b$-field, and dilaton that depend only on time. 
This allowed us to study some generic features for cosmological backgrounds when all $\alpha^{\prime}$ corrections are included. In particular, we discussed solutions such as de Sitter vacua that are nonperturbative in $\alpha^{\prime}$. We close with a list of natural follow-up projects and open problems:

(i) The cosmological backgrounds investigated here involved only a single scale factor $a(t)$ in addition to the dilaton. In general it should be straightforward to include independent "rolling radii" in different dimensions, as analyzed to lowest order in $\alpha^{\prime}$ long ago [29]. Moreover, it would be important to include a $b$-field. Qualitatively new phenomena may appear in this case.

(ii) Eventually it would be crucial to arrive at (semi) realistic cosmological solutions. In particular, one has to make sure that the string coupling given by the dilaton, which here is generally time dependent, does not become too large, for otherwise the classical string theory can no longer be trusted. Moreover, matter fields should be included in a duality covariant fashion, as in [2].

(iii) An important challenge for any theory of quantum gravity is to show how the big-bang singularity could be resolved. (Related work includes [38,39].) One possibility is that the higher-derivative $\alpha^{\prime}$ corrections of classical string theory could resolve the initial singularity, leading to a pre-big-bang phase that is smoothly connected to the post-big-bang phase [32]. With the framework developed here it should be possible to investigate whether this is possible in principle.

(iv) In our classification of higher derivative terms, field redefinitions at each order in $\alpha^{\prime}$ allowed us to eliminate any terms in which the field $\mathcal{S}$ had more than one time derivative or the field $\Phi$ had any number of derivatives. How general is this phenomenon in time-dependent field dynamics?

(v) Arguably a central problem is to determine for each string theory the free coefficients defining $F(H)$, which determines the functions $f(H)$ and $g(H)$ that appear in the $\alpha^{\prime}$-complete Friedmann equations. Perhaps the significantly simplified setup developed here will suggest a viable strategy.

\section{ACKNOWLEDGMENTS}

We are very grateful to Robert Brandenberger, Maurizio Gasperini, Jean-Luc Lehners, Diego Marques, Krzysztof Meissner, Mark Mueller, Ashoke Sen, Andrew Tolley, and Gabriele Veneziano for useful discussions and correspondence. The work of O.H. is supported by the ERC Consolidator Grant "Symmetries \& Cosmology."
[1] R. H. Brandenberger and C. Vafa, Superstrings in the Early Universe, Nucl. Phys. B316, 391 (1989).

[2] A. A. Tseytlin and C. Vafa, Elements of string cosmology, Nucl. Phys. B372, 443 (1992).

[3] M. Gasperini and G. Veneziano, The Pre-big bang scenario in string cosmology, Phys. Rep. 373, 1 (2003).

[4] R. Brandenberger, R. Costa, G. Franzmann, and A. Weltman, Dual spacetime and nonsingular string cosmology, Phys. Rev. D 98, 063521 (2018).

[5] J. Quintin, R. H. Brandenberger, M. Gasperini, and G. Veneziano, Stringy black-hole gas in $\alpha^{\prime}$-corrected dilaton gravity, Phys. Rev. D 98, 103519 (2018).

[6] R. H. Brandenberger, Beyond standard inflationary cosmology, arXiv:1809.04926.

[7] H. Wu and H. Yang, Double field theory inspired cosmology, J. Cosmol. Astropart. Phys. 07 (2014) 024.

[8] D. J. Gross and E. Witten, Superstring modifications of einstein's equations, Nucl. Phys. B277, 1 (1986).

[9] D. J. Gross and J. H. Sloan, The quartic effective action for the heterotic string, Nucl. Phys. B291, 41 (1987).

[10] R. R. Metsaev and A. A. Tseytlin, Order alpha-prime (Two Loop) equivalence of the string equations of motion and the sigma model weyl invariance conditions: Dependence on the dilaton and the antisymmetric tensor, Nucl. Phys. B293, 385 (1987).
[11] W. Siegel, Superspace duality in low-energy superstrings, Phys. Rev. D 48, 2826 (1993).

[12] C. Hull and B. Zwiebach, Double field theory, J. High Energy Phys. 09 (2009) 099.

[13] O. Hohm, C. Hull, and B. Zwiebach, Background independent action for double field theory, J. High Energy Phys. 07 (2010) 016.

[14] O. Hohm, C. Hull, and B. Zwiebach, Generalized metric formulation of double field theory, J. High Energy Phys. 08 (2010) 008.

[15] O. Hohm, W. Siegel, and B. Zwiebach, Doubled $\alpha^{\prime}$-geometry, J. High Energy Phys. 02 (2014) 065.

[16] O. Hohm and B. Zwiebach, Green-Schwarz mechanism and $\alpha^{\prime}$-deformed Courant brackets, J. High Energy Phys. 01 (2015) 012; Double field theory at order $\alpha^{\prime}$, J. High Energy Phys. 11 (2014) 075.

[17] D. Marques and C. A. Nunez, T-duality and $\alpha^{\prime}$-corrections, J. High Energy Phys. 10 (2015) 084.

[18] O. Hohm, U. Naseer, and B. Zwiebach, On the curious spectrum of duality invariant higher-derivative gravity, J. High Energy Phys. 08 (2016) 173.

[19] E. Lescano and D. Marques, Second order higher-derivative corrections in Double Field Theory, J. High Energy Phys. 06 (2017) 104. 
[20] O. Hohm, Background Independence and Duality Invariance in String Theory, Phys. Rev. Lett. 118, 131601 (2017); Background independent double field theory at order $\alpha^{\prime}$ : Metric vs. Frame-like geometry, Phys. Rev. D 95, 066018 (2017).

[21] W. H. Baron, J. J. Fernandez-Melgarejo, D. Marques, and C. Nunez, The Odd story of $\alpha^{\prime}$-corrections, J. High Energy Phys. 04 (2017) 078.

[22] W. H. Baron, E. Lescano, and D. Marqués, The generalized Bergshoeff-de Roo identification, J. High Energy Phys. 11 (2018) 160.

[23] B. Zwiebach, Curvature squared terms and string theories, Phys. Lett. 156B, 315 (1985).

[24] G. Veneziano, Scale factor duality for classical and quantum strings, Phys. Lett. B 265, 287 (1991); K. A. Meissner and G. Veneziano, Symmetries of cosmological superstring vacua, Phys. Lett. B 267, 33 (1991).

[25] A. Sen, $O(d) \times O(d)$ symmetry of the space of cosmological solutions in string theory, scale factor duality and twodimensional black holes, Phys. Lett. B 271, 295 (1991).

[26] K. A. Meissner, Symmetries of higher order string gravity actions, Phys. Lett. B 392, 298 (1997).

[27] T. Kugo and B. Zwiebach, Target space duality as a symmetry of string field theory, Prog. Theor. Phys. 87, 801 (1992).

[28] O. Hohm and B. Zwiebach, T-duality constraints on higher derivatives revisited, J. High Energy Phys. 04 (2016) 101.

[29] M. T. Mueller, Rolling radii and a time dependent dilaton, Nucl. Phys. B337, 37 (1990).

[30] O. Hohm and B. Zwiebach, Non-perturbative de Sitter vacua via $\alpha^{\prime}$ corrections, arXiv:1905.06583.
[31] G. Arciniega, P. Bueno, P. A. Cano, J. D. Edelstein, R. A. Hennigar, and L. G. Jaime, Geometric inflation, arXiv: 1812.11187.

[32] M. Gasperini, M. Maggiore, and G. Veneziano, Towards a nonsingular pre-big-bang cosmology, Nucl. Phys. B494, 315 (1997).

[33] J. Maharana and J. H. Schwarz, Noncompact symmetries in string theory, Nucl. Phys. B390, 3 (1993).

[34] O. Hohm, A. Sen, and B. Zwiebach, Heterotic effective action and duality symmetries revisited, J. High Energy Phys. 02 (2015) 079.

[35] H. Hata, Soft dilaton theorem in string field theory, Prog. Theor. Phys. 88, 1197 (1992); O. Bergman and B. Zwiebach, The Dilaton theorem and closed string backgrounds, Nucl. Phys. B441, 76 (1995); P. Di Vecchia, R. Marotta, and M. Mojaza, Soft behavior of a closed massless state in superstring and universality in the soft behavior of the dilaton, J. High Energy Phys. 12 (2016) 020.

[36] H. S. Wilf, Lectures on Integer Partitions, https://www.math .upenn.edu/ wilf/PIMS/PIMSLectures.pdf.

[37] H. Yang and B. Zwiebach, Rolling closed string tachyons and the big crunch, J. High Energy Phys. 08 (2005) 046.

[38] T. Biswas, A. Mazumdar, and W. Siegel, Bouncing universes in string-inspired gravity, J. Cosmol. Astropart. Phys. 03 (2006) 009.

[39] T. Biswas, T. Koivisto, and A. Mazumdar, Towards a resolution of the cosmological singularity in non-local higher derivative theories of gravity, J. Cosmol. Astropart. Phys. 11 (2010) 008. 